\title{
Convex projective structures on Gromov-Thurston manifolds
}

\author{
MiCHAEL KAPOVICH
}

\begin{abstract}
We study Gromov-Thurston examples of negatively curved $n$-manifolds which do not admit metrics of constant sectional curvature. We show that for each $n>3$ some of the Gromov-Thurston manifolds admit strictly convex real-projective structures.
\end{abstract}

53C $15,53 \mathrm{C} 20 ; 20 \mathrm{~F} 06$

\section{Introduction}

Gromov and Thurston in [10] constructed, for each $n \geq 4$, examples of compact $n-$ manifolds which admit metrics of negative curvature, with arbitrarily small pinching constants, but do not admit metrics of constant curvature. We review these examples in Section 3. The main goal of this paper is to put convex projective structures on GromovThurston examples. Suppose that $\Omega \subset \mathbb{R} P^{n}$ is an open subset and $\Gamma \subset P G L(n+1, \mathbb{R})$ is a subgroup acting properly discontinuously on $\Omega$. The quotient orbifold $Q=\Omega / \Gamma$ has the natural projective structure $c$. The structure $c$ is said to be (strictly) convex iff $\Omega$ is a (strictly) convex proper subset of $\mathbb{R} P^{n}$. In this case we refer to $Q$ as (strictly) convex projective orbifold.

Our main result then is the following theorem.

Theorem 1.1 Gromov-Thurston examples admit strictly convex projective structures.

We refer the reader to Section 7 for the more precise statement. Our theorem will be proven in Section 7 via "bending" of the original hyperbolic structure on a certain hyperbolic manifold $M$ (used to construct Gromov-Thurston examples) in the manner similar to [10], where flat-conformal structures were constructed on certain negatively curved manifolds.

There are two parts in this proof: (1) Producing a projective structure, (2) proving that the structure is convex. Then strict convexity of the structure follows from the Benoist's theorem below (Theorem 1.2), since Gromov-Thurston examples have Gromov-hyperbolic fundamental groups. 
Part (1) is dealt with by solving a certain product of matrices problem, which is a special case of a Lie-theoretic problem interesting on its own right, see Section 5. The projective manifolds $M^{\prime}$ are then built by gluing convex subsets of the hyperbolic manifolds $M$. By passing to the universal cover we obtain a tessellation of $\tilde{M}^{\prime}$ by convex polyhedra in $\mathbb{Q}^{n}$, each of which has infinitely many facets.

Dealing with (2) is especially interesting, since, at present, there is only one general method for proving convexity of projective structures, namely via Vinberg-Tits fundamental domain theorem [17]. Unfortunately, this theorem applies only to reflection groups, which cannot be used in higher dimensions. Our approach to proving convexity is to adapt Vinberg's arguments in a more general context of manifolds obtained by gluing convex cones with infinitely many faces. In this setting, Vinberg's arguments (requiring polyhedrality of the cones) do not directly apply and we modify them by appealing to the small cancellation theory, see Section 4.

Remark J Lee recently proved a generalization of the fundamental domain theorem for projective structures coming from a certain class of compact convex polyhedra not covered by Vinberg-Tits theorem, see [14]. It is interesting to note that while one of the key geometric ingredients of our proof is the geometry of $C A T(0)$ spaces, the proof of $\mathrm{J}$ Lee uses the geometry of Alexandrov spaces (with curvature $\geq 1$ ).

The main motivation for this paper comes from the following beautiful theorem.

Theorem 1.2 (Y Benoist, [2]) Suppose that a convex projective orbifold $M$ is compact. Then $M$ is strictly convex iff $\Gamma=\pi_{1}(M)$ is Gromov-hyperbolic.

Examples of convex-projective structures on compact orbifolds are provided by the quotients of round balls in $\mathbb{R} P^{n}$ by discrete cocompact groups of automorphisms. The Hilbert metric on such examples is a Riemannian metric of constant negative sectional curvature. Thus such orbifolds are hyperbolic. By deforming the above examples in $\mathbb{R} P^{n}$ one obtains other examples of strictly convex projective manifolds/orbifolds.

In 2002 I was asked by Bruce Kleiner and Francois Labourie if one can construct examples of compact strictly convex projective manifolds which are not obtained by deforming hyperbolic examples. The main goal of this paper is to prove that such examples indeed exist in all dimensions $\geq 4$. Independently, such examples were constructed by Yves Benoist in dimension 4 using reflection groups, see [3]. The paper [3] also produces "exotic" strictly convex subsets $\Omega$ in $\mathbb{R} P^{n}$ for all $n \geq 3$ : The metric space $\left(\Omega, d_{H}\right)$ is Gromov-hyperbolic but is not quasi-isometric to $\mathbb{H}^{n}$, where $d_{H}$ is the Hilbert metric on $\Omega$. However these examples do not appear to admit discrete cocompact groups of automorphisms. 
Organization of the paper In Section 2 we review the basics of convex projective structures; we also prove a relative rigidity result for certain hyperbolic manifolds with corners. In Section 3 we describe Gromov-Thurston examples in details and prove that they are not homotopy-equivalent to hyperbolic manifolds.

In Section 4 we prove the main technical result of this paper, a convexity theorem for a certain class of projective structures. It is used to prove convexity of the projective structures on Gromov-Thurston examples. In Section 5, we use hyperbolic geometry in order to solve an algebraic "product of matrices" problem, which is the algebraic ingredient of the proof of Theorem 1.1. In Section 6 we describe the bending deformations of projective structures. These deformations are used in order to convert the algebraic results of Section 5 into projective structures on Gromov-Thurston examples. In Section 7 we put everything together and prove Theorem 1.1.

Acknowledgments During the work on this paper I was partially supported by the NSF grant DMS-04-05180. I am grateful to John Millson for explaining to me the construction of arithmetic examples in Section 3, to Yves Benoist and Bruce Kleiner for useful conversations and to Martin Bridson for several remarks concerning geometry and topology of the complex $Z$ used in this paper. I am also grateful to the referee for several useful suggestions.

\section{Geometric preliminaries}

\subsection{Projective structures}

Let $X$ be a real-analytic $n$-manifold and $G \curvearrowright X$ be a real-analytic Lie group action. An $(X, G)$-structure on an $n$-manifold $M$ is a maximal atlas $A=\left\{\left(U_{i}, \phi_{i}\right): i \in I\right\}$ where the $U_{i}$ are open subsets of $M$ and $\phi_{i}: U_{i} \rightarrow \phi_{i}\left(U_{i}\right) \subset X$ are charts, so that the transition maps

$$
\phi_{j} \circ \phi_{i}^{-1}
$$

are restrictions of elements of $G$. Every $(X, G)$-structure on $M$ determines a pair

$$
(\operatorname{dev}, \rho)
$$

where dev: $\tilde{M} \rightarrow X$ is a local homeomorphism defined on the universal cover of $M$ and $\rho: \pi_{1}(M) \rightarrow G$ is a representation so that $\operatorname{dev}$ is $\rho$-equivariant. The map $\mathrm{dev}$ is called the developing map and $\rho$ is called the holonomy representation of $A$. Conversely, each pair (dev, $\rho$ ), where $\rho$ is a homomorphism $\pi_{1}(M) \rightarrow G$ and $\operatorname{dev}$ is a $\rho$-equivariant local homeomorphism, determines an $(X, G)$-structure on $M$. 
Remark Analogous definitions make sense for orbifolds.

Clearly, every open subset $\Omega \subset X$ has a canonical $(X, G)$-structure can induced from $X$. If $\Gamma \subset G$ acts properly discontinuously and freely on $\Omega$ then can projects to the quotient manifold $\Omega / \Gamma$.

The most relevant examples of $(X, G)$-structures for this paper are:

(1) (Real) projective structures, where $X=\mathbb{R} P^{n}, G=P G L(n+1, \mathbb{R})$ is the group of projective transformations.

(2) Affine structures, where $X=\mathbb{R}^{n}, G=G L(n, \mathbb{R}) \ltimes \mathbb{R}^{n}$ is the group of affine transformations.

Clearly, every affine structure is also projective. Conversely, given any projective structure on $M^{n}$ there is a canonical affine structure on the appropriate line bundle over $M^{n}$, induced by the tautological line bundle over $\mathbb{R} P^{n}$.

We refer the reader to Goldman [8; 9] and Choi-Goldman [5] for the foundational material on real-projective structures.

\subsection{Convex sets}

For a subset $E \subset \mathbb{R} P^{n}$, let $\operatorname{Span}(E)$ denote the smallest subspace in $\mathbb{R} P^{n}$ containing $E$. We will use the notation $\bar{E}$ to denote the closure of a subset $E$ of a topological space.

A subset $K \subset \mathbb{R}^{n+1}$ is called a convex homogeneous cone if it is convex and is invariant under multiplication by positive numbers. The cone $K$ is proper if $K \neq \mathbb{R}^{n+1}$.

A subset $C \subset \mathbb{R} P^{n}$ is said to be convex if it is the image of a convex homogeneous cone $\widehat{C} \subset \mathbb{R}^{n+1} \backslash\{0\}$ under the projection

$$
\text { proj: } \mathbb{R}^{n+1} \backslash\{0\} \rightarrow \mathbb{R} P^{n} .
$$

An open subset $C \subset \mathbb{R} P^{n}$ is convex if and only there exists a linear subspace $\mathbb{R} P^{n-1} \subset$ $\mathbb{R} P^{n}$ such that $C$ is a convex subset of the affine space $\mathbb{A}^{n}=\mathbb{R} P^{n} \backslash \mathbb{R} P^{n-1}$.

Suppose that $\Omega$ is an open convex subset of $\mathbb{R} P^{n}$. Then $\Omega$ is said to be strictly convex if its frontier contains no nondegenerate segments.

Given a point $x \in \mathbb{R}^{n}$ and a set $B \subset \mathbb{R}^{n}$, let $\Sigma=$ Cone $_{x}(B)$ denote the union of all segments $\overline{x b}, b \in B$. We will refer to $x$ as the tip and $B$ as the base of this cone.

Lemma 2.1 If $B$ is convex then $\Sigma$ is also convex. 
Proof Let $p, q \in \Sigma$. Then there exist $a, b \in B$ such that $p \in \overline{a x}, q \in \overline{b x}$. Thus the segment $\overline{p q}$ is contained in the planar triangle $\Delta(a, b, x)$ with the vertices $a, b, x$. Since $\overline{a b} \subset B$, it follows that

$$
\overline{p q} \subset \Delta(a, b, x) \subset \Sigma .
$$

Lemma 2.2 Let $C \subset \mathbb{R}^{n}$ be an open convex subset, $x \in \bar{C}$ and $y \in C$. Then the half-open interval $(x, y]:=\overline{x y} \backslash\{x\}$ is contained in $C$.

Proof Let $B \subset C$ denote an open round ball centered at $y$ and let $\Sigma=$ Cone $_{x}(B)$ denote the cone with the tip at $x$ and base $B$, which is the union of the segments connecting $x$ to the points of $B$. Then the open segment $(x, y)$ is contained in the interior $\Sigma^{0}$ of $\Sigma$. By convexity, $\Sigma \subset \bar{C}$. Hence $(x, y) \subset \Sigma^{0} \subset C$.

Suppose that $A$ is a projective structure on an $n$-manifold $M$. Let $\widetilde{A}$ denote the lift of $A$ to the universal cover $\tilde{M}$ of $M$.

Definition 2.3 The projective structure $A$ is called convex if dev: $(\tilde{M}, \tilde{A}) \rightarrow \mathbb{R} P^{n}$ is an isomorphism onto a convex subset in $\mathbb{R} P^{n}$.

In other words, convex projective structures appear as quotients $\Omega / \Gamma$, where $\Omega \subset \mathbb{R} P^{n}$ is convex and $\Gamma$ is a properly discontinuous group of projective transformations of $\Omega$.

\subsection{Relative rigidity}

The results of this section will be used in the proof of Theorem 3.3. The key problem discussed here is a special case of the following relative version of Mostow Rigidity and Calabi Local Rigidity.

\section{Problem 1}

(1) Let $\left(X, g_{0}\right)$ be a compact convex hyperbolic manifold of dimension $n \geq 3$. Suppose that $g_{t}$ is a continuous family of hyperbolic metrics on $X$ so that all the manifolds $\left(X, g_{t}\right)$ are convex hyperbolic manifolds and the induced path metric on $\partial X$ is constant. Does it follows that all manifolds $\left(X, g_{t}\right)$ are isometric?

(2) Suppose that $X_{1}, X_{2}$ are compact convex hyperbolic manifolds of dimension $n \geq 3$ and

$$
h:\left(X_{1}, \partial X_{1}\right) \rightarrow\left(X_{2}, \partial X_{2}\right)
$$

is a homotopy-equivalence of pairs, which induces an isometry of the boundaries with respect to the induced path metric. Does it follow that $h$ is homotopic to an isometry $X_{1} \rightarrow X_{2}$ ? 
The problem is closely related to Kapovich [11, Problem 11.8]. To the best of my knowledge, both parts of Problem 1 are open even for $n=3$. We will consider this problem in the special setting of manifolds with corners defined below.

Definition 2.4 Let $(X, g)$ be a metrically complete hyperbolic $n$-manifold with convex boundary. We will say that $(X, g)$ is a convex hyperbolic manifold with codimension 2 geodesic corners if the following holds. The boundary $\partial X$ is the union $Y^{+} \cup Y^{-}$, where $Y^{ \pm}$are compact totally-geodesic submanifolds of dimension $n-1$, so that $Y^{+} \cap Y^{-}=Z$ is an $n-2$-dimensional totally-geodesic submanifold. The submanifold $Z$ will be called the corner in $X$. See Figure 1.

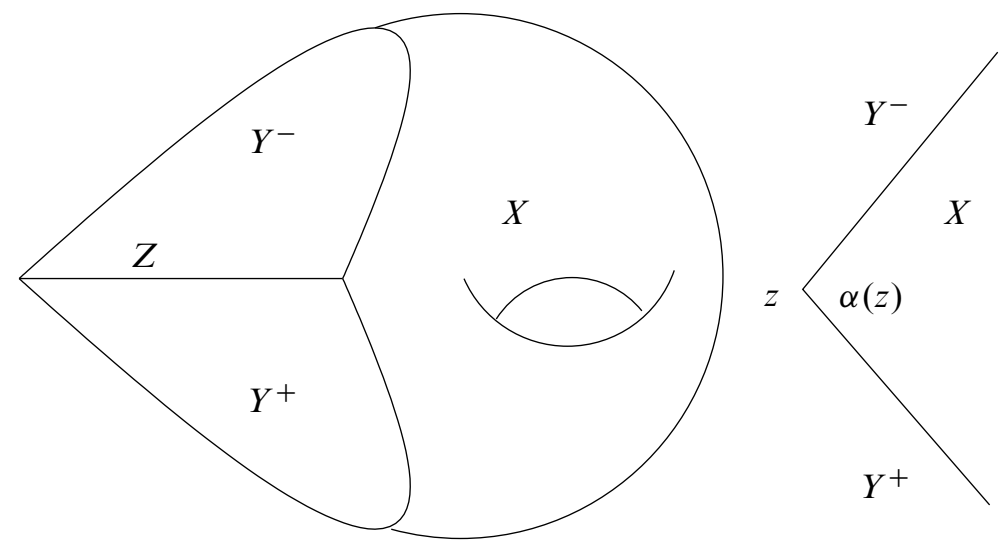

Figure 1: Manifold with codimension 2 corners.

We then obtain the induced path metric $g \mid \partial X$ on the boundary of $X$. Clearly, the induced metric is hyperbolic. At each point $z \in Z$ we have the dihedral angle $\alpha(z)$ at which $Y^{+}$meets $Y^{-}$along $Z$. The dihedral angle $\alpha(z)$ is locally constant along $Z$. To simplify the notation we will frequently omit the metric $g$ and will simply say that $X$ itself is a convex hyperbolic manifold with codimension 2 geodesic corners. Given two such manifolds $X_{1}, X_{2}$, a proper isometry

$$
\partial X_{1} \rightarrow \partial X_{2}
$$

is an isometry of the induced metrics which carries $Z_{1}$ to $Z_{2}$. We will say that $X$ is symmetric if it admits an isometric involution $\tau: X \rightarrow X$ which fixes $Z$ pointwise and interchanges $Y^{+}$and $Y^{-}$.

One can prove the following relative version of Calabi's Local Rigidity Theorem (although we do not need it for this paper). 
Theorem 2.5 Let $X$ be a compact manifold of dimension $\geq 3, g_{t}$ is a continuous family of hyperbolic metrics on $X$ so that all the manifolds $\left(X, g_{t}\right)$ are convex hyperbolic manifolds with codimension 2 geodesic corner $Z$ (which does not depend on the metric) and the induced path metrics on $\partial X$ are all isometric. Then all the manifolds $\left(X, g_{t}\right)$ are isometric.

For the purposes of the proof of Theorem 3.3, we will need the following simple relative version of the Mostow Rigidity Theorem.

Lemma 2.6 Let $n \geq 3$. We assume that $X_{1}, X_{2}, X_{3}$ are symmetric $n$-dimensional compact convex hyperbolic manifold with codimension 2 geodesic corners, so that the dihedral angles at the corners of $X_{1}, X_{2}, X_{3}$ are constant and equal to

$$
\alpha_{1}=\frac{\pi}{m}, \quad \alpha_{2}=\frac{\pi}{m-1}, \quad \alpha_{3}=\frac{2 \pi}{2 m-1}
$$

respectively, where $m \in \mathbb{Z}, m \geq 2$. Then there cannot exist a pair of homotopyequivalences

$$
f_{13}:\left(X_{1}, \partial X_{1}\right) \rightarrow\left(X_{3}, \partial X_{3}\right), \quad f_{23}:\left(X_{2}, \partial X_{2}\right) \rightarrow\left(D_{3}, \partial X_{3}\right)
$$

which restrict to proper isometries on the boundaries of these manifolds.

Proof The idea of the proof is to construct two homotopy-equivalent non-isometric hyperbolic $n$-manifolds $W^{\prime}, W^{\prime \prime}$ by gluing copies of $X_{1}, X_{2}, X_{3}$ in two different ways and then obtain a contradiction with the Mostow Rigidity Theorem.

(1) We construct $W^{\prime}$ by gluing $2 m-1$ copies of $X_{3}$ (the manifolds $X_{3} \times\{i\}$, $i=1, \ldots, 2 m-1)$ via isometries between the copies of $Y_{3}^{+}$and $Y_{3}^{-}$. Here we are using the fact that $X_{3}$ is symmetric; therefore we have the isometry from $Y_{3}^{+} \times\{i\}$ to $Y_{3}^{-} \times\{i+1\}$. See Figure 2. The resulting manifold is a connected compact manifold which is clearly hyperbolic away from the image $Z^{\prime}$ of the union of corners

$$
\bigcup_{i=1}^{2 m} Z_{3} \times\{i\} .
$$

Since the total angle along $Z^{\prime}$ is

$$
(2 m-1) \frac{2 \pi}{2 m-1}=2 \pi
$$

the manifold $W^{\prime}$ is hyperbolic. We let $Y_{i}^{\prime}, i=1, \ldots, 2 m-1$ denote the submanifolds with boundary in $W^{\prime}$, which are the images of

$$
Y_{3}^{+} \times\{i\}, i=1, \ldots, 2 m-1 \text {. }
$$



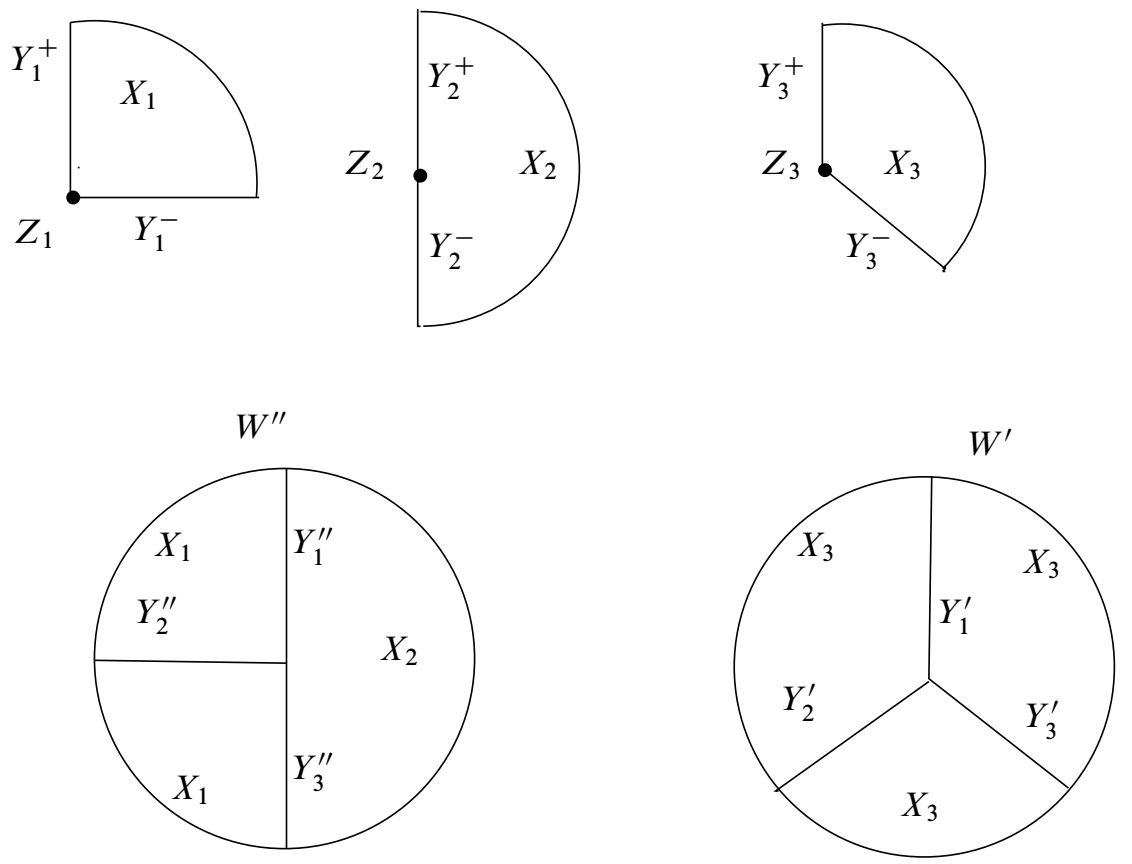

Figure 2: Gluing manifolds $W^{\prime}, W^{\prime \prime}$ in the case $m=2$.

Thus $Z^{\prime \prime}=\partial Y_{i}^{\prime}$ for each $i$.

(2) We take $m$ copies of $X_{1}, m-1$ copies of $X_{2}$ and glue them together using isometries interchanging $Y_{i}^{ \pm}, i=1,2$, as in Figure 2. As in Part 1, the resulting manifold $W^{\prime \prime}$ is hyperbolic since

$$
m \frac{\pi}{m}+(m-1) \frac{\pi}{m-1}=2 \pi .
$$

We let $Z^{\prime \prime} \subset W^{\prime \prime}$ denote the image of the union of corners of the manifolds $X_{1} \times\{i\}, X_{2} \times\{j\}$. Let $Y_{i}^{\prime \prime}$ denote the submanifolds with boundary in $W^{\prime \prime}$ which are the images of

$$
Y_{1}^{+} \times\{i\}, i=1, \ldots, m, \quad Y_{2}^{+} \times\{i\}, i=m+1, \ldots, 2 m-1 .
$$

Define a map

$$
f: W^{\prime \prime} \rightarrow W^{\prime}
$$


by using the maps

$$
\begin{aligned}
& f_{13} \times\{i\}: X_{1} \times\{i\} \rightarrow X_{3} \times\{i\}, i=1, \ldots, m, \\
& f_{23} \times\{i\}: X_{2} \times\{i\} \rightarrow X_{3} \times\{i\}, i=m+1, \ldots, 2 m-1 .
\end{aligned}
$$

We leave it to the reader to verify that we get a homotopy-equivalence $f: W^{\prime \prime} \rightarrow W^{\prime}$. By the construction, $f\left(Z^{\prime \prime}\right)=Z^{\prime}$ and

$$
f\left(Y_{i}^{\prime \prime}\right)=Y_{i}^{\prime}, i=1, \ldots, 2 m-1 .
$$

By Mostow Rigidity, there is an isometry $\phi: W^{\prime \prime} \rightarrow W^{\prime}$ homotopic to $f$. Since $Y_{i}^{\prime}, Z^{\prime}, Y_{i}^{\prime \prime}, Z^{\prime \prime}$ are totally-geodesic, it follows that

$$
\phi\left(Z^{\prime \prime}\right)=Z^{\prime}, \quad \phi\left(Y_{i}^{\prime \prime}\right)=Y_{i}^{\prime}, i=1, \ldots, 2 m-1 .
$$

Therefore for each $i$, the dihedral angles between $Y_{i}^{\prime \prime}, Y_{i+1}^{\prime \prime}$ are equal to the dihedral angles between $Y_{i}^{\prime}, Y_{i+1}^{\prime}$. The former angles however are equal to $\alpha_{3}$ and the latter are equal to $\alpha_{2}$ or $\alpha_{3}$ respectively. Contradiction.

We now show that Lemma 2.6 can be improved provided that $n \geq 4$. The requirement that $n \geq 4$ stems from the fact that our proof uses the Mostow Rigidity Theorem in dimension $n-1$.

Lemma 2.7 Let $n \geq 4$. We assume that $X_{1}, X_{2}$ are symmetric $n$-dimensional compact convex hyperbolic manifolds with codimension 2 geodesic corners, so that the dihedral angles at the corners of $X_{1}, X_{2}$ are constant and equal to

$$
\alpha_{1}=\frac{\pi}{m}, \quad \alpha_{2}=\frac{\pi}{m-1}
$$

respectively, where $m \in \mathbb{Z}, m \geq 2$. Then there is no proper homotopy-equivalence

$$
f_{12}:\left(X_{1}, \partial X_{1}\right) \rightarrow\left(X_{2}, \partial X_{2}\right) .
$$

Proof Suppose that the homotopy-equivalence $f_{12}$ exists. Since the boundaries of $X_{1}, X_{2}$ (with the induced hyperbolic metrics) are compact hyperbolic $n-1$-manifolds and $n-1 \geq 3$, it follows from Mostow rigidity that $f_{12}$ is homotopic to a proper homotopy-equivalence which is an isometry of the boundaries of these manifolds. We retain the notation $f_{12}$ for the new homotopy-equivalence.

We will use the manifolds $X_{1}, X_{2}$ to construct a third manifold with corners $X_{3}$ so that $X_{1}, X_{2}, X_{3}$ satisfy the assumptions of Lemma 2.6.

Take $m$ isometric copies of the manifold $X_{1}$ :

$$
X_{1} \times\{i\}, 1 \leq i \leq m,
$$


and $m-1$ isometric copies of the manifold $X_{2}$ :

$$
X_{2} \times\{i\}, m+1 \leq i \leq 2 m-1,
$$

and glue them together similarly to the proof of Lemma 2.6. Since

$$
m \frac{\pi}{m}+(m-1) \frac{\pi}{m-1}=2 \pi,
$$

the resulting manifold $M$ is a compact connected hyperbolic $n$-manifold. The homotopy-equivalence $f_{12}: X_{1} \rightarrow X_{2}$ yields a homotopy-action

$$
\eta: \mathbb{Z}_{2 m-1} \curvearrowright M
$$

which cyclically permutes the above copies of $X_{1}$ and $X_{2}$. We now use the assumption that $X_{1}$ is symmetric (the earlier arguments were only using the symmetry of the boundaries of $X_{1}$ and $X_{2}$ ). Let $\tau_{1}: X_{1} \rightarrow X_{1}$ be an isometric reflection fixing $Z_{1}$, the fundamental domain for $\left\langle\tau_{1}\right\rangle$ is a manifold with corners $X_{4}$ which "half" of $X_{1}$;

$$
\partial X_{4}=Y_{4}^{+} \cup Y_{4}^{-}
$$

where $Y_{4}^{+}=Y_{1}^{+}$.

Since all the "pieces" of $M$ were properly homotopy-equivalent to $X_{1}$, it follows that the homotopy-action $\eta$ extends to a homotopy-action of the dihedral group

$$
\eta: D_{2 m-1} \curvearrowright M \text {. }
$$

A "fundamental domain" for this action is the image of $X_{4}$ in the appropriate copy of $X_{1}$ in $M$. By abusing the notation, we again denote this image by $X_{1}$. By applying the Mostow Rigidity Theorem (in dimension $n$ ) we replace the above homotopy-action with an isometric action

$$
\zeta: D_{2 m-1} \curvearrowright M \text {. }
$$

The fixed hypersurfaces for the reflections in $\zeta\left(D_{2 m-1}\right) \subset I \operatorname{som}(M)$, of course, are not the same as the fixed hypersurfaces for the corresponding reflections in $\eta\left(D_{2 m-1}\right)$, but they are homotopic to each other. Therefore we can choose a fundamental domain for the action $\zeta$ to be a manifold with corners $X_{5} \subset M$, so that

$$
\begin{aligned}
\partial X_{5} & =Y_{5}^{+} \cup Y_{5}^{-}, \\
\pi_{1}\left(X_{5}\right) & =\pi_{1}\left(X_{4}\right) \subset \pi_{1}(M), \\
\pi_{1}\left(Y_{5}^{ \pm}\right) & =\pi_{1}\left(Y_{4}^{ \pm}\right) \subset \pi_{1}(M) .
\end{aligned}
$$

We let $\sigma \in D_{2 m-1}$ be such that $\zeta(\sigma)$ is an isometric reflection fixing $Y_{5}^{-}$and set

$$
X_{3}:=X_{5} \cup \zeta(\sigma)\left(X_{5}\right) .
$$


Then $X_{3}$ is a compact hyperbolic $n$-manifold with geodesic corners,

$$
\partial X_{3}=Y_{5}^{+} \cup \zeta(\sigma)\left(Y_{5}^{+}\right)=Y_{3}^{+} \cup Y_{3}^{-} .
$$

The corner $Z_{3}$ of $X_{3}$ is homotopic to the corner of $X_{5}$. Clearly, the angle along $Z_{3}$ equals

$$
\frac{2 \pi}{2 m-1} \text {. }
$$

Since $X_{4}$ is properly homotopy-equivalent to $X_{5}$ (with homotopy-equivalence preserving the corners), we obtain a proper homotopy-equivalence

$$
h: X_{1} \rightarrow X_{3} \text {. }
$$

The boundary $\partial X_{3}$ of $X_{3}$ (with the induced metric) is a hyperbolic $n-1$-manifold, which is properly homotopy-equivalent to the hyperbolic $n-1$-manifold $\partial X_{1}$. Therefore we can homotop $h$ to another proper homotopy-equivalence $f_{13}: X_{1} \rightarrow X_{3}$, which restricts to an isometry of the boundaries of these manifolds. We set

$$
f_{23}:=f_{13} \circ f_{21}
$$

where $f_{21}$ is the homotopy-inverse to $f_{12}: X_{1} \rightarrow X_{2}$. Without loss of generality we may assume that $f_{21} \mid \partial X_{2}=\left(f_{12} \mid \partial X_{1}\right)^{-1}$ and, therefore, is an isometry. Thus $f_{23}$ restricts to an isometry $\partial X_{2} \rightarrow \partial X_{3}$.

The triple $X_{1}, X_{2}, X_{3}$ satisfies all the assumptions of Lemma 2.6. Therefore we obtain a contradiction with that Lemma 2.6.

\section{Gromov-Thurston examples}

In this section we review Gromov-Thurston examples [10] of compact $n$-manifolds (more generally, orbifolds) which admit metrics of negative curvature but do not admit metrics of constant curvature. Note that Gromov and Thurston [10] have other examples as well. These examples will not be discussed here.

Consider the quadratic form

$$
\varphi(x)=x_{1}^{2}+\cdots+x_{n}^{2}-\sqrt{p} x_{n+1}^{2}
$$

where $p$ is a (positive) prime number, and $n \geq 2$. Let $\widetilde{\Gamma}=\operatorname{Aut}(\varphi) \cap G L(n+1, \mathbb{Z})$; then $\widetilde{\Gamma}$ is a cocompact arithmetic subgroup in $\operatorname{Aut}(\varphi) \cong O(n, 1)$.

We let $H$ denote the Lorentzian model of the hyperbolic space $\mathbb{H}^{n}$ :

$$
\left\{x: \varphi(x)=-1, x_{n+1}>0\right\} .
$$


Consider the linear subspace

$$
V=\left\{x \in \mathbb{R}^{n+1}: x_{1}=x_{2}=0\right\} .
$$

The intersection $V \cap H$ is a totally-geodesic codimension 2 hyperbolic subspace. The stabilizer of $V$ in $\widetilde{\Gamma}$ acts cocompactly on $V \cap H$ since it is isomorphic to the set of integer points in the algebraic group

$$
\operatorname{Aut}\left(x_{3}^{2}+\cdots+x_{n}^{2}-\sqrt{p} x_{n+1}^{2}\right) .
$$

Suppose that $W \subset \mathbb{R}^{n+1}$ is a rational codimension 1 linear subspace containing $V$. Then the Lorentzian (with respect to $\varphi$ ) involution $\tau_{W}$ fixing $W$ pointwise belongs to $G L(n+1, \mathbb{Q})$. Thus the groups $\widetilde{\Gamma}$ and $\tau_{W} \widetilde{\Gamma} \tau_{W}$ are commensurable. Therefore, there exists a finite index subgroup $\Gamma_{W} \subset \widetilde{\Gamma}$ which is normalized by $\tau_{W}$. By applying this procedure to two appropriately chosen rational hyperplanes $W^{+}, W^{-}$passing through $V$ we obtain the following lemma.

Lemma 3.1 Given a number $m \geq 1$ there exists a subgroup $\hat{\Gamma} \subset \operatorname{Aut}(\varphi)$ commensurable to $\widetilde{\Gamma}$, which contains a dihedral subgroup $D_{m}$ fixing $V$ pointwise. The generating involutions in $D_{m}$ acts as reflections.

By passing to an appropriate torsion-free normal subgroup $\Gamma \subset \hat{\Gamma}$ we get a compact hyperbolic manifold $M=H / \Gamma$. Let $\bar{\Gamma}$ denote the subgroup of $\hat{\Gamma}$ generated by $\Gamma$ and the dihedral subgroup $D_{m}$.

The group $D_{m}$ acts on $M$ isometrically with a fundamental domain $O$ (that can be identified with the orbifold $M / D_{m}=\mathbb{H}^{n} / \bar{\Gamma}$ ), which is a manifold with corners so that the corner (which is possibly disconnected) corresponds to the hyperbolic subspace $V \cap \mathbb{H}^{n}$. Then we can think of the manifold $M$ as obtained by gluing $2 m$ copies of $X$. The dihedral angle at the corner is $\pi / m$. By abusing the notation we will keep the notation $V$ for the corner of $M$.

The boundary of $O \backslash V$ is the union of two codimension 1 totally-geodesic (possibly disconnected) submanifolds. We denote the closures of these submanifolds $Y^{+}, Y^{-}$ (they correspond to the hyperplanes $W^{+}, W^{-} \subset \mathbb{H}^{n}$ ); then $V=Y^{+} \cap Y^{-}$.

Assumption 2 We assume from now on that the manifold $M$ admits an isometric action $D_{2 m} \curvearrowright M$ of a dihedral group $D_{2 m}$ which contains $D_{m}$ as an index 2 subgroup and fixes $V$.

Therefore there is an isometric involution $\sigma: O \rightarrow O$ which fixes $V$ and interchanges $Y^{+}$and $Y^{-}$. We now construct new a manifold (without boundary) $M^{\prime}$ by gluing $2 m-2$ copies of $O$, cf Section 2.3. 

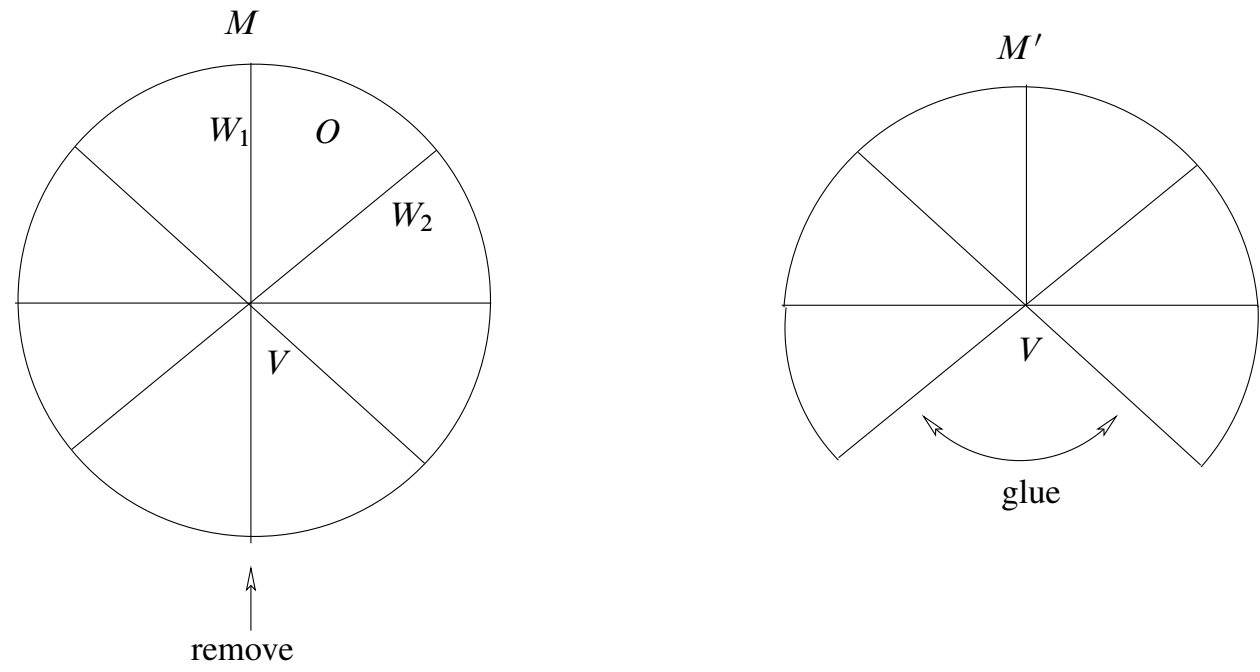

Figure 3: Constructing the manifold $M^{\prime}$.

Remark Another class of Gromov-Thurston examples is obtained by gluing $2 m+2$ copies of $O$. Construction of convex projective structures on such manifolds is possible, but is a bit more complicated than the one explained in this paper, therefore these manifolds will not be discussed here.

We will think of $M, M^{\prime}$ as doubles of the manifolds $N, N^{\prime}$ which are obtained by gluing $m, m-1$ copies of $O$ respectively. Thus $M^{\prime}$ is obtained by "subtracting" two copies of $O$ from $M$.

In Section 7 we will use an alternative description of $M^{\prime}$. Assumption 2 implies that the manifold $N^{\prime}$ admits a reflection symmetry $\theta^{\prime}$ fixing the submanifold $V$. Then $M^{\prime}$ is diffeomorphic to the manifold obtained by gluing two copies of $N^{\prime}$ via the involution $\theta^{\prime} \mid \partial N^{\prime}$ of the boundary.

Proposition 3.2 (Gromov, Thurston, [10]) For every sufficiently large $m$, the manifold $M^{\prime}$ admits a metric of negative sectional curvature varying in the interval

$$
\left[-1+\epsilon_{m},-1\right]
$$

Moreover, $\lim _{m \rightarrow \infty} \epsilon_{m}=0$

Remark Note that $M^{\prime}$ admits a canonical singular Riemannian metric which is smooth and hyperbolic away from $V$. The negatively curved Riemannian metric on 
$M^{\prime}$ is obtained by modifying the above singular metric on a regular $R$-neighborhood of $V$. This modification works provided that $R$ is sufficiently large, which is achieved by taking large $m$. Alternatively, one can fix $m$ and pass to an appropriate finite-index subgroup of $\Gamma$.

Thus $\pi_{1}\left(M^{\prime}\right)$ is Gromov-hyperbolic provided that $m$ is sufficiently large.

Theorem 3.3 (Gromov-Thurston [10]) If $n \geq 4$ then $M^{\prime}$ is not homotopy-equivalent to a closed manifold of constant (negative) sectional curvature.

Proof Our proof is a variation of the argument given in [10]. By construction, the hyperbolic manifold $M$ is the union of $m$ hyperbolic manifolds with codimension 2 geodesic corners isometric to a fixed manifold with corners $X_{1}:=O$. The angle at the corner of $O$ equals $\pi / m$. Suppose that $M^{\prime}$ is homotopy-equivalent to a closed hyperbolic manifold $M^{\prime \prime}$. The group $D_{m-1}$ acts topologically on $M^{\prime}$ so that the fundamental domain is homeomorphic to the manifold with corners $O$. By the Mostow Rigidity Theorem, there exists an isometric action $D_{m-1} \curvearrowright M^{\prime \prime}$ and an equivariant homotopy equivalence

$$
f: M^{\prime} \rightarrow M^{\prime \prime} .
$$

By equivariance, $f$ projects to a homotopy-equivalence

$$
h: M^{\prime} / D_{m-1} \rightarrow M^{\prime \prime} / D_{m-1}
$$

which preserves the strata of the singular loci of the corresponding orbifolds. We identify the quotients with the fundamental domains $O$ and $O^{\prime \prime}$ for the corresponding actions of the reflection groups $D_{m-1} \curvearrowright M^{\prime}, D_{m-1} \curvearrowright M^{\prime \prime}$. Thus, $X_{2}=O^{\prime \prime}$ is a manifold with codimension 2 corners properly homotopy-equivalent to $X_{1}=O$. The corner of $O^{\prime \prime}$ is fixed by this action of $D_{m-1}$ on $M^{\prime \prime}$. Therefore the angle at the corner equals $\pi /(m-1)$.

It remains to verify that the hyperbolic manifolds with corners $X_{1}$ and $X_{2}$ are symmetric. By Assumption 2, the isometric action $D_{m} \curvearrowright M$ extends to an isometric action $D_{2 m} \curvearrowright M$. Therefore a generating involution of $D_{2 m}$ determines a symmetry $\sigma$ of $X_{1}$ which fixes the corner. Hence $X_{1}$ is symmetric. The symmetry $\sigma$ yields a symmetry of the fundamental domain of $D_{m-1} \curvearrowright M^{\prime}$. Therefore the topological action $D_{m-1} \curvearrowright M^{\prime}$ extends to a topological action $D_{2(m-1)} \curvearrowright M^{\prime}$. The latter, in turn, yields an isometric action $D_{2(m-1)} \curvearrowright M^{\prime \prime}$ extending the isometric action $D_{m-1} \curvearrowright M^{\prime \prime}$. Hence the fundamental domain $O^{\prime \prime}=X_{2}$ of $D_{m-1}$ is also symmetric.

The existence of the proper homotopy-equivalence

$$
\left(X_{1}, \partial X_{1}\right) \rightarrow\left(X_{2}, \partial X_{2}\right)
$$


contradicts Lemma 2.7.

Definition 3.4 When $n \geq 4$ (and $m$ is sufficiently large), we will refer to the manifolds $M^{\prime}$ as Gromov-Thurston examples.

\section{A convexity theorem}

In geometry one frequently constructs geometric objects by gluing together other geometric objects. For instance, given hyperbolic $n$-manifolds $M_{1}, M_{2}$ with totally geodesic boundaries and an isometry $\phi: \partial M_{1} \rightarrow \partial M_{2}$, one constructs a new hyperbolic manifold $M=M_{1} \cup_{\phi} M_{2}$ by gluing $M_{1}$ and $M_{2}$ via $\phi$. Under some mild assumptions, if $M_{1}, M_{2}$ are both complete, then so is $M$. (For instance, it suffices to assume that the boundaries of both $M_{1}, M_{2}$ have positive normal injectivity radii.) Another instance of this phenomenon is Poincare's fundamental domain theorem, where instead of gluing manifolds with boundary one glues manifolds with corners.

Recall that in a complete connected Riemannian manifold any two points can be connected by a geodesic. Therefore, the most natural generalization of the notion of completeness in the category of projective structures is convexity. The problem however is that typically, union of convex sets is not convex. Therefore we have to impose further restrictions in order to get convexity of projective manifolds obtained by gluing other projective manifolds.

Below is a simple example (which I owe to Yves Benoist) of failure of convexity of an affine structure built out of convex fundamental domains.

Let $P$ denote the convex 2-dimensional polygon in $\mathbb{R}^{2}$ with the vertices

$$
(1,0),(2,0),(0,1),(0,2) \text {. }
$$

Let $A(x)=2 x$ and $B$ be the rotation by the angle $\pi / 2$. By gluing the sides of $P$ via $A$ and $B$ we obtain an affine structure on the torus. However this structure is not convex since the image of the developing map is $\mathbb{R}^{2} \backslash\{0\}$.

The main result of this section is a version of Poincare's fundamental domain theorem in the context of convex projective structures. We will show that, under some conditions, an affine manifold $X$ obtained by linear gluing of convex homogeneous cones (with infinitely many faces) is again convex. Projectivizing this statement we get a similar result for projective structures.

One can also regard this result as a combination theorem for groups of linear transformations (preserving the above cones). The classical Klein-Maskit combination 


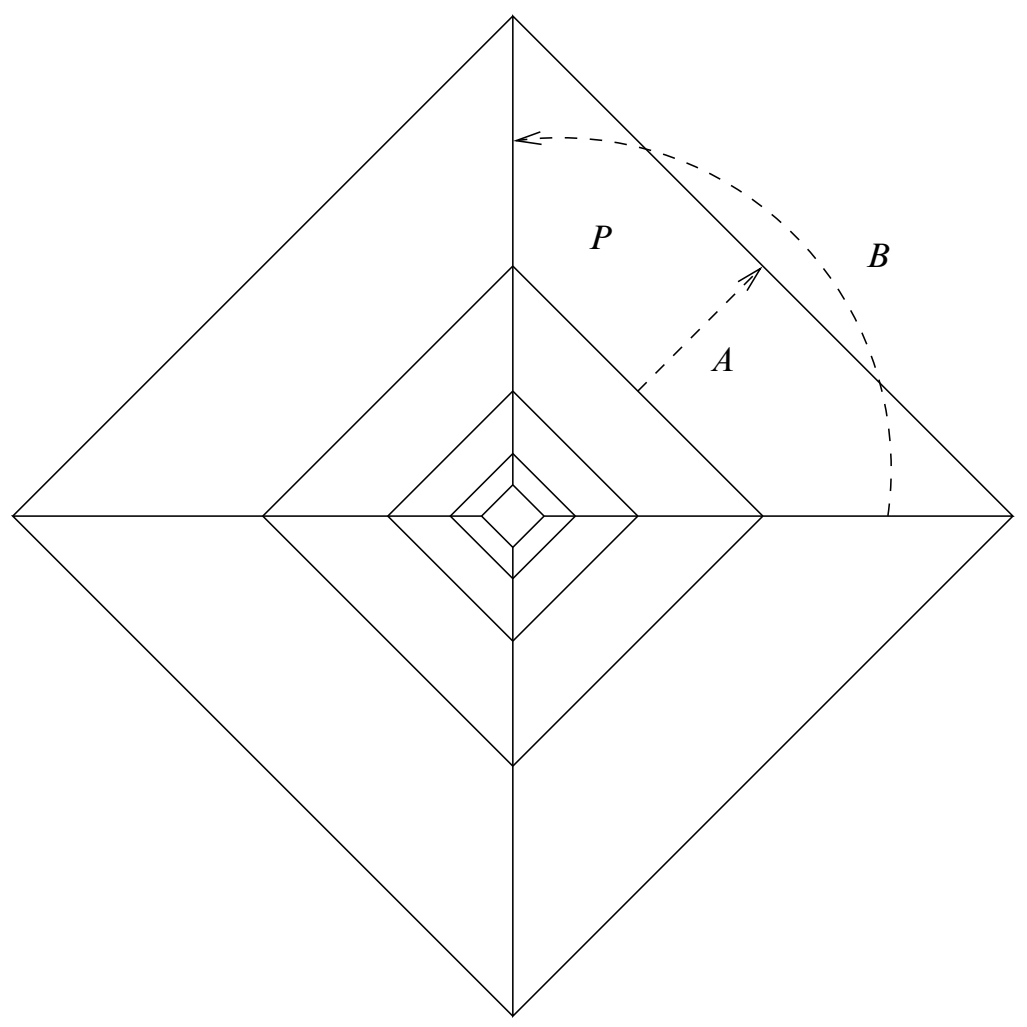

Figure 4: Convex fundamental domain $P$ for a nonconvex projective structure.

theorems deal with the fundamental groups of graphs of groups $G=\pi_{1}(\mathcal{G})$ and the combinatorial structure behind these combination theorems is that of a simplicial tree. Geodesic paths in this tree correspond to the normal forms of the elements of $G$. In contrast, we are dealing with the fundamental groups of 2-dimensional complexes of groups; accordingly, the combinatorial structure of the stratification of $X$ is encoded in a 2-dimensional cell complex $Z$. The normal forms that we will be using are represented by local geodesics in the 1 -skeleton of $Z$. In our analysis of the local geodesics we are helped by the fact that $Z$ satisfies a strong form of a certain small cancellation condition; this enables us to describe all local geodesics connecting given pair of vertices of $Z$.

For the rest of this section we will assume that $C$ is an open proper convex homogeneous cone in $\mathbb{R}^{n}$. 


\section{Definition 4.1}

(1) An (open) facet of $C$ is a maximal open convex homogeneous $(n-1)$-dimensional cone contained in the boundary of $C$.

(2) A codimension $k$ face of $C$ is a maximal open convex $(n-k)$-dimensional cone in $\bar{C}$, which is contained in the boundary of $C$ provided that $k \neq 0$.

We will use the notations $\bar{F}, \bar{C}$, etc to denote the closures in $\mathbb{R}^{n}$ of faces, cones, etc. Accordingly, we will refer to closed facets, closed codimension $k$ faces of $C$, etc.

Let $\mathcal{F}$ be a certain collection of faces of $F$ of codimension $\leq 2$, so that $C \in \mathcal{F}$. We define a new convex cone $C^{\prime}$ as

$$
C^{\prime}=\bigcup_{F \in \mathcal{F}} F
$$

Then $C \subset C^{\prime} \subset \bar{C}$. For a face $F \in \mathcal{F}$ we let $F^{\prime}$ denote the closure of $F$ in $C^{\prime}$. By abusing the notation we will continue to refer to the sets $F^{\prime}$ as faces of $C^{\prime}$.

For each face $F$ of $C$ define the limit set

$$
\Lambda(F):=\bar{F} \backslash F^{\prime} .
$$

Assumption 3 We assume that $C$ and $\mathcal{F}$ are such that:

(1) Each face $F \in \mathcal{F}$ of codimension $\neq 0$ is the intersection of the higher-dimensional faces $H_{i}^{\prime} \in \mathcal{F}$.

(2) For each pair of distinct facets $F_{1}, F_{2} \in \mathcal{F}$, either $\bar{F}_{1} \cap \bar{F}_{2}=\{0\}$ or

$$
\bar{F}_{1} \cap \bar{F}_{2} \backslash\{0\}=F_{1}^{\prime} \cap F_{2}^{\prime}
$$

is a codimension 2 face $F \in \mathcal{F}$.

(3) For each facet $F \in \mathcal{F}$ we require $\Lambda(F)$ to have empty interior in $\partial F$. Therefore, for every $x \in \mathbb{R}^{n}$, the cone $\Sigma_{x}(\Lambda(F))$ does not locally separate $\mathbb{R}^{n}$.

(4) We require that for each point $x \in \Lambda(C)$ there exists a sequence of distinct facets $F_{j} \in \mathcal{F}$ and points $x_{j} \in F_{j}$ so that $\lim _{j} x_{j}=x$. In particular, the cell $C$ has infinitely many facets and hence $n \geq 3$.

(5) $\Lambda(C)$ is closed.

The motivation for this list of assumptions comes from the following example which will appear in Section 7. Suppose that the projectivization of $C^{\prime}$ is isomorphic to a closed convex subset $H \subset \mathbb{U}^{n-1}$. (Here we use the projective model for the hyperbolic 
space.) Suppose that $G$ is a discrete subgroup of $\operatorname{Isom}\left(\mathbb{T}^{n-1}\right) \subset P G L(n, \mathbb{R})$ which preserves $H$ and such that $H / G$ is compact.

Then the projectivization $\operatorname{proj}(\Lambda(C) \backslash\{0\})$ of $\Lambda(C)$ is the limit set of the group $G$. Therefore the limit set $\Lambda(C)$ satisfies (4) and (5). Moreover, for each facet $F \subset C^{\prime}$, the limit set $\operatorname{proj}(\Lambda(F))$ is the limit set of the stabilizer of $\operatorname{proj}(F)$ in $G$. Therefore $C$ also satisfies (2) and (3), cf Susskind and Swarup [16].

In fact, the hyperbolic manifold with corners $H / G$ will be the manifold $O$ which appeared in Section 3. The faces of the cone $C$ will correspond to the totally-geodesic boundary strata of $O$. In particular, all the faces will have codimension $\leq 2$. This explains the part 1 of Assumption 3.

Suppose now that $X$ is an affine $n$-manifold obtained by gluing countably many copies $C_{j}^{\prime}, j \in J$, of the cone $C^{\prime}$ via linear isomorphisms of facets $F^{\prime}$ of $C^{\prime}$. We will refer to the cones $C_{j}$ as cells in $X$. Cells whose intersection is a facet are called adjacent. Strata of $X$ are the the faces $F_{i}^{\prime}$ of cones $C_{j}$. We refer to this partition of $X$ into strata as the stratification of $X$.

Assumption 4 We assume that:

(1) For each point in every codimension 2 face $E$ of $C_{j} \subset X$, the tessellation of $X$ by the adjacent cells is locally isomorphic to a tessellation of $\mathbb{R}^{n}$ by cones cut off by a family of $t$ hyperplanes passing through a codimension 2 subspace, so that $4 \leq t<\infty$. (Thus the number of cones containing $E$ is $2 t \in[8, \infty)$.) See Figure 5 .

(2) For every pair of cells $C_{i}^{\prime}, C_{j}^{\prime}$ sharing a facet $F$, the union $C_{i}^{\prime} \cup C_{j}^{\prime}$ is convex.

(3) $X$ simply-connected.

Note that this assumption is satisfied in a number of important cases, eg for tessellations corresponding to linear reflection groups and in the example which appears in Section 7.

In a similar fashion we define a cell complex $\bar{X}$ by extending the above gluing maps to the closed cells $\bar{C}_{j}$. The origin $0 \in \bar{X}$ is the point corresponding to 0 in the closed cone $C$. We give $X$ and $\bar{X}$ the quotient topology with respect to the projection

$$
\bigsqcup_{j \in J} C_{j} \rightarrow X, \quad \bigsqcup_{j \in J} \bar{C}_{j} \rightarrow \bar{X}
$$

Since $X$ contains infinitely many cells, the space $\bar{X}$ is not locally compact (the origin is incident to infinitely many cells). 


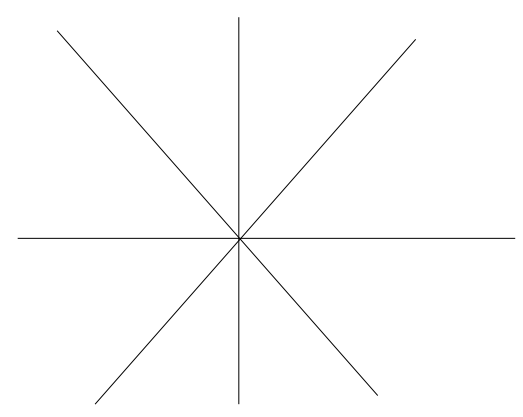

Figure 5: In this example $t=4$.

Remark More generally, one can allow spaces $X$ built out of non-isomorphic convex cones $C_{j}$. The argument used in our proof still apply in this case, but we do not need this for the purposes of this paper.

We will refer to the closed faces $F^{\prime}$ of $X$ as the strata of $X$. Hence $X$ becomes a stratified space with the strata of codimension 0,1 and 2 .

We then have a developing map dev: $X \rightarrow \mathbb{R}^{n}$, which is a linear isomorphism on each cell $C_{j}$. The developing map extends naturally to a continuous map dev: $\bar{X} \rightarrow \mathbb{R}^{n}$. This map determines the notion of a segment $[x, y]$ in $\bar{X}$, which is defined as a path which is mapped by dev homeomorphically to a straight-line segment in $\mathbb{R}^{n}$.

Definition 4.2 A subset $G$ of $\bar{X}$ is called convex if every two points in $G$ can be connected by a segment contained in $G$.

A wall $H$ in $X$ is a maximal connected subset of $X$ which is the union of facets $F_{j}^{\prime}$ so that each point $x \in H$ has a neighborhood $U \subset H$ mapped by dev homeomorphically to an open disk (or a half-disk) of a hyperplane in $\mathbb{R}^{n}$. Thus the developing map sends each wall to a subset of a hyperplane in $\mathbb{R}^{n}$. Therefore, a segment $\sigma \subset X$ can intersect a wall $H$ transversally in at most one point.

Remark One can show that every wall is a manifold without boundary.

The main result of this section is the following theorem.

Theorem 4.3 Suppose that $X$ satisfies Assumption 3 and Assumption 4. Then:

(1) $\bar{X}$ is convex and dev: $\bar{X} \rightarrow \mathbb{R}^{n}$ is a continuous bijection onto a convex homogeneous cone in $\mathbb{R}^{n}$. 
(2) $X$ is convex and the developing map dev is a homeomorphism of $X$ onto a proper open convex homogeneous cone in $\mathbb{R}^{n}$.

Proof We first sketch the main steps of the proof. Our proof is modelled on the standard arguments appearing in the proofs of Poincaré's fundamental domain theorem (cf Vinberg [17]).

Before studying geometry of the spaces $X$ and $\bar{X}$, we analyze the combinatorial structure of these complexes. Our main tool is the small cancellation theory for the 2 -dimensional cell complex $Z$ below which encodes this combinatorics. (The $q-$ dimensional cells in $Z$ correspond to the codimension $q$ strata in the stratification of $X$ and the inclusion relation in $Z$ is the reverse inclusion with respect to the relation between the strata of $X$.) The main results of this combinatorial analysis are:

(1) Proposition 4.8 which establishes convexity and compactness of the unions of geodesics $g e o(v, w)$ in $Z^{(1)}$ connecting vertices $v, w \in Z^{(0)}$.

(2) Lemma 4.11 which shows that generic line segments $[x, y] \subset X$ project to geodesic paths in $Z^{(1)}$.

The geodesic paths in $Z^{(1)}$ then correspond to minimal galleries in $\bar{X}$. Such galleries are chains of cells

$$
C_{0}^{\prime} \cup \cdots \cup C_{k}^{\prime}
$$

in $X$ so that the consecutive cells share a facet. Minimality of such a gallery means that it has the least length (ie the number of cells) among all galleries connecting $C_{0}^{\prime}$ to $C_{k}^{\prime}$.

The key for proving Theorem 4.3 is to show that the union of closed minimal galleries $\overline{\operatorname{Gal}}(A, B)$ connecting cells $A$ and $B$ is convex in $\bar{X}$. This is done by induction on the length of the galleries. The induction step is based on Proposition 4.12 which, roughly speaking, claims that the set $Y=Y(x)$ of points $y \in B$ which can be connected to a point $x \in A$ by a segment $[x, y] \subset \overline{\operatorname{Gal}}(A, B)$ is both closed and open. The actual statement of the proposition is somewhat more complicated. Proof of the closedness of $Y$ is a direct corollary of finiteness of the number of cells in $\overline{\operatorname{Gal}}(A, B)$, which, in turn, follows from compactness of geo $(v, w)$. Proof of openness of $Y$ is less obvious. If the segment $[x, y] \subset \overline{\operatorname{Gal}}(A, B)$ is contained in $\operatorname{Gal}(A, B)=\overline{\operatorname{Gal}}(A, B) \cap X$, then the existence of $\left[x, y^{\prime}\right] \subset \operatorname{Gal}(A, B)$ for $y^{\prime}$ near $y$ follows from the fact that $\operatorname{Gal}(A, B)$ is a locally convex subset of the affine manifold $X$ (cf Lemma 2.2). The main difficulty in the proof stems from the fact that the segment $[x, y]$ can (a priori) pass through the points of $\bar{X} \backslash X$. 
Once convexity of $\bar{X}$ is established, the proof of convexity of $X$ is essentially a topological argument. We prove (Lemma 4.9) that for every $W=\overline{\operatorname{Gal}}(A, B)$, the complement $W \backslash X$ is contained in the boundary of $W$. Therefore, by Lemma 2.2, for $x \in A$ and $y \in B^{\prime}$ the segment $[x, y]$ is contained in $X$. The proof in the case when $x \in A^{\prime}, y \in B^{\prime}$ requires a more complicated argument.

We now begin the proof of Theorem 4.3. Let $Z$ denote the complex dual to the stratification of $X$ into faces. The vertices of $Z$ are the cells in $X$. Each facet $F$ in $X$ yields an edge $e \subset Z$ connecting the vertices corresponding to the cells adjacent to $F$. Each codimension 2 face $E$ in $X$ yields a 2-cell in $Z$, whose edges correspond to the facets in $X$ containing $E$.

Then $Z$ is a regular 2-dimensional cell complex, in particular, nonempty intersections between cells in $Z$ are again cells. Each 2 -face $c$ of $Z$ is a $2 t$-gon for a certain $t \geq 4$ (depending on $c$ ). Part 4 of Assumption 3 implies that each vertex of $Z$ is contained in infinitely many edges.

We will equip $Z$ with the weak topology. According to Corollary A.2 in the Appendix, $Z$ is simply-connected.

Let $T:=Z^{(1)}$ be the 1 -skeleton of $Z$. We define a path metric $d$ on $T$ by declaring each edge of this graph to have the unit length. We also metrize $Z$ by declaring each facet of $Z$ to be isometric to the regular Euclidean polygon with sides of the unit length. This yields a path metric $\rho$ on $Z$. Since $Z$ is not locally finite, the topology determined by $\rho$ does not agree with the weak topology on $Z$. Nevertheless, according to a theorem of Dowker [6], the map

$$
Z \rightarrow(Z, \rho)
$$

is a homotopy-equivalence. In particular, $(Z, \rho)$ is simply-connected.

Since $C$ and its facets are convex it follows that the links of vertices of $Z$ contain no bigons. Therefore $Z$ satisfies the following properties

\section{Property 5}

(1) Each 2 -cell in $Z$ has at least 7 edges. (In fact, at least 8 edges.)

(2) For every vertex $v$ of $Z$, the combinatorial length of each embedded circle in the link of $v$ is at least 3 .

Therefore $Z$ satisfies the small cancellation condition $C^{\prime}(1 / 7)$, where the pieces are the edges of $T$, see Ghys and de la Harpe [7, Appendix]. The same properties also imply that $(Z, \rho)$ is a $C A T(0)$ metric space, see Bridson and Haefliger [4]. Thus $Z$ is contractible. The main difficulty is that $Z$ is not locally finite. This is where we deviate from Vinberg's proof [17]. 


\subsection{Local geodesics in $T$}

Definition 4.4 A path $p$ in $T$ is a local geodesic (or Dehn-reduced) if it contains no backtracks and no subpaths of length $>t$ contained in a single $2 t$-gonal $2-$ cell of $Z$.

The main result of this section is Proposition 4.8. It proves that the union $\operatorname{geo}_{\text {loc }}(x, y)$ of all local geodesics in $T$ connecting vertices $x$ and $y$, is a finite subgraph in $T$, which is strongly convex. For every pair of vertices $z, w$ in $\operatorname{geo}_{\text {loc }}(x, y)$, every local geodesic connecting $z$ and $w$ is contained in $\operatorname{geo}_{\text {loc }}(x, y)$.

A degenerate bigon in $T$ has two vertices $x, y$ and two equal edges $\alpha, \beta$, which are local geodesics in $T$.

Given two vertices $x, y \in T$ which belong to a common $2 t$-gonal 2-cell $c$ of $Z$ and which are distance $t$ apart, there are exactly two geodesics $\alpha, \beta \subset T$ (of the length $t$ ) connecting $x$ to $y$.

More generally, define a corridor $K$ in $Z$ as a union of 2 -cells $c_{1}, \ldots, c_{l}(l \geq 1)$ of the complex $Z$ so that for each $i$ :

(1) $c_{i}, c_{i+1}$ share an edge $e_{i}$, called an interior edge of the corridor $K$.

(2) The edges $e_{i-1}, e_{i}$ are "antipodal" on the boundary of $c_{i}$.

Thus each corridor $K$ yields a wall in $X$. Consider the topological circle $\lambda$ which is the boundary of the corridor $K=c_{1} \cup \cdots \cup c_{l}$. Pick two vertices $x, y \in \lambda$ which break $\lambda$ in the union of two local geodesics $\beta, \gamma$ connecting $x$ and $y$. (Note that $x$ and $y$ are not uniquely determined by $K$.)

Definition 4.5 In case both $\beta, \gamma$ are local geodesics, we refer to $\beta \cup \gamma$ as a simple bigon with the vertices $x, y$. We will say that $K$ connects the vertices $x$ and $y$.

Since $Z$ satisfies the condition $C^{\prime}(1 / 7)$, according to [7, Proposition 39, Part (i)], all local geodesic bigons $B=\beta \cup \gamma$ in $T$ are concatenations of degenerate bigons and simple bigons. See Figure 6.

Remark The proofs in [7, Appendix] are given under the assumption that the cell complex is the Cayley complex of a finitely-presented group. However the proofs needed for [7, Proposition 39, Part (i)] do not require this assumption.

We will think of the bigon $B$ together with the union of corridors bounded by the simple sub-bigons in $B$ as a (Van Kampen) diagram $D$ embedded in $Z$ so that the boundary of $D$ is $B$. (To be more precise, one has to take the reverse of the path $\gamma$ here but we will ignore this issue.) As usual, we define the $\operatorname{area}, \operatorname{Area}(B):=\operatorname{Area}(D)$, to be the number of the 2-cells which appear in $D$. 


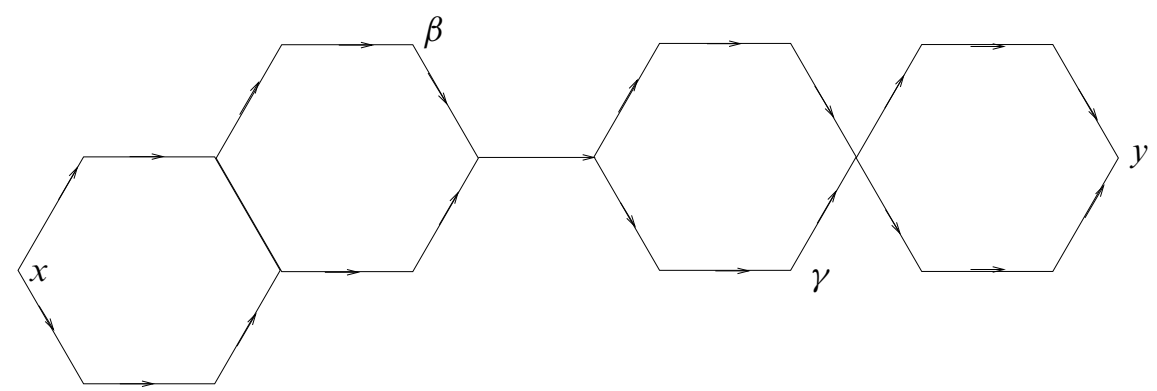

Figure 6: A geodesic bigon with the vertices $x, y$, which is the union of three simple bigons and one degenerate bigon.

Remark Since $Z$ is 2-dimensional and contractible, it is easy to see that the diagram $D$ is the least area disk bounded by the bigon $B$.

We let $\overline{a b} \subset(Z, \rho)$ denote the geodesic segment connecting the points $a$ and $b$. We observe that for each corridor $K$ connecting vertices $x$ and $y$, the segment $\overline{x y}$ is contained in $K$. Moreover, the intersection of $\overline{x y}$ with the boundary of $K$ is finite. It therefore follows that a corridor connecting $x$ and $y$ is unique.

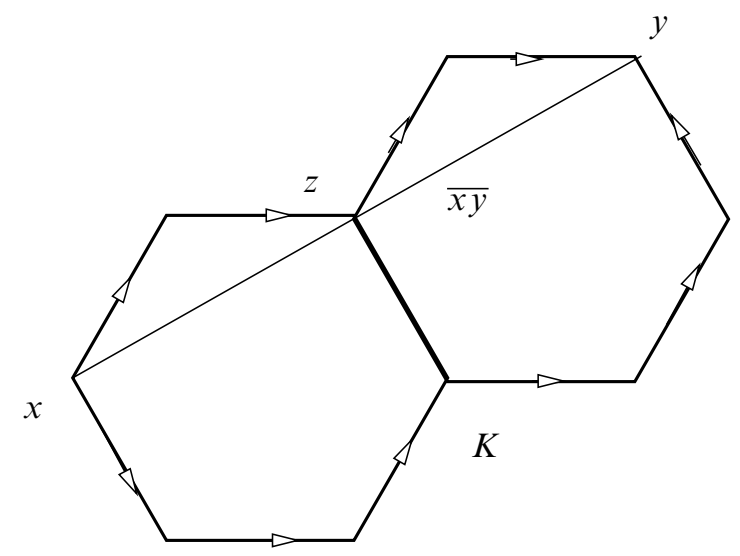

Figure 7: A corridor $K$ with the vertices $x, y$, so that $\overline{x y} \cap \partial K=\{x, y, z\}$.

Claim 4.6 Suppose that $B=\alpha \cup \beta$ is a bigon with vertices $x$ and $y$ which bounds a corridor $D$. Then for every pair of vertices $z \in \alpha, w \in \beta$ such that $d(z, w)>1$ we have:

For every corridor $K$ connecting $z$ and $w, K$ shares a 2-cell with $D$. 
Proof Under the above assumptions, the geodesic segment $\overline{z w} \subset Z$ has nonempty intersection with the interior of a $2-$ cell $c \subset D$. Since the complement $\overline{z w} \backslash \operatorname{int}(K)$ is finite, it follows that $c \subset K$.

\section{Lemma 4.7}

(1) Let $B=\alpha \cup \beta$ be a bigon with vertices $x$ and $y$. Then

$$
\operatorname{Area}(B) \leq \frac{1}{2} \min (\text { length }(\alpha), \text { length }(\beta)) .
$$

(2) length $(\alpha) \leq 2$ length $(\beta)$.

(3) length $(\alpha) \leq 2 d(x, y)$.

(4) $\operatorname{Area}(B) \leq d(x, y)$.

\section{Proof}

(1) For every 2-cell $c_{i}$ in the diagram $D$ bounded by $B$, let $\alpha_{i}:=\alpha \cap c_{i}, \beta_{i}:=\beta \cap c_{i}$. Then

$$
\min \left(\text { length }\left(\alpha_{i}\right), \text { length }\left(\beta_{i}\right)\right) \geq 2 .
$$

This immediately implies (1).

(2) For each $c_{i}$, length $\left(\alpha_{i}\right)+$ length $\left(\beta_{i}\right) \geq \operatorname{length}\left(\partial c_{i}\right)-2$ and

$$
\max \left(\text { length }\left(\alpha_{i}\right), \text { length }\left(\beta_{i}\right)\right) \leq \frac{1}{2} \text { length }\left(\partial c_{i}\right) .
$$

Therefore

$$
\mid \text { length }\left(\alpha_{i}\right)-\text { length }\left(\beta_{i}\right) \mid \leq 2 \text {. }
$$

Since

$$
\min \left(\text { length }\left(\alpha_{i}\right), \text { length }\left(\beta_{i}\right)\right) \geq 2,
$$

we obtain

$$
\text { length }\left(\alpha_{i}\right) \leq 2 \text { length }\left(\beta_{i}\right) .
$$

The assertion (2) follows.

(3) Let $\gamma$ be a geodesic in $T$ connecting $x$ to $y$. By applying Part 2 to the bigon $\alpha \cup \gamma$, we obtain

$$
\text { length }(\alpha) \leq 2 \text { length }(\gamma)=2 d(x, y) .
$$

(4) By combining (1) and (3) we obtain

$$
\operatorname{Area}(B) \leq \frac{1}{2} \text { length }(\alpha) \leq d(x, y) .
$$

Proposition 4.8 Let $x, y \in T$, be vertices so that $d(x, y)=d$. Then there exist diagrams $D, D_{\text {loc }} \subset Z$ bounded by bigons $B_{\text {loc }}, B$ with vertices $x, y$ so that: 
(1) The union $\operatorname{geo}_{\mathrm{loc}}(x, y)$ of all local geodesics connecting $x$ to $y$ is equal to $D_{\mathrm{loc}}^{(1)}$. The union geo $(x, y)$ of all geodesics connecting $x$ to $y$ is equal to $D^{(1)}$. In particular, there are only finitely many local geodesics connecting $x$ and $y$.

(2) The set geo $(x, y)$ is convex in $T$. The set $\operatorname{geo}_{\mathrm{loc}}(x, y)$ is strongly convex, ie, every local geodesic connecting vertices in $\mathrm{geo}_{\mathrm{loc}}(x, y)$ is contained in $\operatorname{geo}_{\mathrm{loc}}(x, y)$.

(3) The distance between any two vertices in $\operatorname{geo}(x, y)$ is $\leq d$. In particular, $\operatorname{diam}(\operatorname{geo}(x, y))=d$.

Proof Since by Lemma 4.7, the bigons connecting $x$ and $y$ have area $\leq d(x, y)$, there exists a (locally geodesic) bigon $B_{\text {loc }}=\alpha \cup \beta$ connecting $x$ and $y$ and having maximal area among such bigons. Let $D_{\text {loc }}$ denote the diagram in $Z$ bounded by $B_{\text {loc }}$. We leave it to the reader to verify that $D_{\text {loc }}^{(1)}$ is contained in $\operatorname{geo}_{\mathrm{loc}}(x, y)$. It is done by examining the corridors in $D_{\text {loc }}$.

We now claim that for each pair of vertices $z, w$ in $D_{\text {loc }}$,

$$
\operatorname{geo}_{\mathrm{loc}}(z, w) \subset D_{\text {loc }} .
$$

This will prove both $\mathrm{geo}_{\mathrm{loc}}(x, y)=D_{\mathrm{loc}}^{(1)}$ and strong convexity of $\mathrm{geo}_{\mathrm{loc}}(x, y)$. Moreover, it will show that the number of local geodesics in $\operatorname{geo}_{\text {loc }}(x, y)$ is finite, since the cell complex $D_{l o c}$ is finite.

Suppose that there exists a local geodesic $\gamma \subset T$ connecting $z$ and $w$, which is not contained in $D_{\text {loc }}$. Without loss of generality, we may assume that $\gamma \cap D_{\text {loc }}=\{z, w\}$ (otherwise we replace $\gamma$ with its subpath).

Case $1 z, w$ belong to $\alpha$. Let $\alpha_{x z}, \alpha_{z w}, \alpha_{w y}$ denote the subpaths of $\alpha$ connecting $x$ and $z, z$ and $w, w$ and $y$ respectively. The bigon

$$
B^{\prime}:=\gamma \cup \alpha_{z w}
$$

bounds a diagram $D^{\prime} \subset Z$ whose interior is disjoint from $D_{\text {loc }}$. Set

$$
\alpha^{\prime}:=\alpha_{x z} \cup \gamma \cup \alpha_{w y}
$$

Then the bigon $\alpha^{\prime} \cup \beta$ with the vertices $x, y$, bounds the diagram $D_{\text {loc }} \cup D^{\prime}$ whose area is greater than $\operatorname{Area}\left(D_{\text {loc }}\right)$. Contradiction.

Case $2 z$ and $w$ are separated by a point in $D_{\text {loc }}$. In this case we replace $\alpha$ and $\beta$ by appropriately chosen (local) geodesics $\alpha^{\prime}, \beta^{\prime} \subset D^{(1)}$ which still bound $D_{\text {loc }}$ so that $z, w \in \alpha^{\prime}$. Then we are done by Case 1 . 
Case $3 z, w$ belong to the same corridor $K \subset D_{\text {loc }}$. Without loss of generality we may assume that $d(z, w)>1$ (otherwise the local geodesic connecting $z$ and $w$ is unique and is contained in $D_{\text {loc }}^{(1)}$ ). Then there exists a local geodesic $\delta \subset K^{(1)}$ connecting $z$ to $w$. The bigon

$$
B^{\prime}=\gamma \cup \delta
$$

bounds a diagram $K^{\prime} \subset Z$. This diagram has to be a corridor, since, otherwise, the intersection $\gamma \cap K$ consists of more than two points. By Claim 4.6, the intersection $K \cap K^{\prime}$ contains a 2-cell $F$. Since $\gamma \cap F$ consists of at least one edge, we obtain a contradiction with our assumption on the intersection $\gamma \cap C$.

Therefore geo $_{\text {loc }}$ is strongly convex.

In order to define the diagram $D$ we observe that some of the simple bigons $B^{\prime}$ whose concatenation is $B_{l o c}$ are not geodesic. One of the sides of $B^{\prime}$ is geodesic while the other is not. Therefore we construct $B$ by replacing each non-geodesic simple bigon in $B_{l o c}$ with its geodesic side. Let $D$ denote the diagram bounded by $B$. Since $\mathrm{geo}_{\text {loc }}$ is strongly convex, for every pair of vertices $z, w \in D^{(1)}$, every local geodesic $\gamma$ connecting $z$ and $w$ is contained in $D_{\mathrm{loc}}^{(1)}$. If $\gamma$ is geodesic, then, by the construction of $D$, it follows that $\gamma \subset D^{(1)}$. Therefore $D^{(1)}$ is convex.

This proves the first two assertions of Proposition. In order to prove the third assertion observe that if $z, w \in D^{(1)}$ do not belong to a common corridor, then each geodesic $[z, w] \subset D^{(1)}$ connecting $z$ to $w$ can be extended to a geodesic connecting $x$ and $y$. Therefore in this case $d(z, w) \leq d(x, y)$.

Suppose that $z, w$ belong to a common corridor $C \subset D$. Then $d(x, y)$ is at least $h$, which is half of the perimeter of $C$. On the other hand, $z, w$ subdivide the boundary of $C$ in two arcs. The length of the shorter of these arcs is at most $h$. Therefore $d(z, w) \leq$ $h \leq d(x, y)$. Finally, $x, y \in \operatorname{geo}(x, y)$ and $d(x, y)=d$. Thus diam $(\operatorname{geo}(x, y))=d$. Thus the proposition is proved.

Remark Clearly, the proof of the above proposition used only the fact that $Z$ is simply-connected and satisfies Property 5.

\subsection{Segments in $X$, galleries and geodesics in $T$}

In this section we relate segments in $X$ and local geodesics in the graph $T$. The main result here is that generic segments in $X$ correspond to geodesics in $T$.

Lemma 4.9 Consider the union $W \subset \bar{X}$ of finitely many closed cells $\bar{C}_{i}, i=1, \ldots, k$. Then $\operatorname{int}(W) \subset X$. In particular, 0 is never in the interior of $W$. 
Proof Since two closed cells can share at most one facet, the set $W$ projects to a finite subgraph $p(W) \subset T$. The complement $W \backslash X$ is the union

$$
\bigcup_{i=1}^{k} \Lambda\left(C_{i}\right) \text {. }
$$

Pick a point $x \in \Lambda\left(C_{i}\right)$. Then, according to Part 4 of the Assumption 3, there exists an infinite sequence of facets $F_{j} \subset \partial C_{i}$ and points $x_{j} \in F_{j}$ such that

$$
x=\lim _{j} x_{j} .
$$

Since the graph $p(W)$ contains only finitely many edges, only a finite number of the facets $F_{j}$ are contained in a cell in $W$ different from $C_{i}$. Therefore all but finitely many of $x_{j}$ 's belong to the boundary of $W$, which implies that $x$ cannot belong to the interior of $W$.

Definition 4.10 Let $q$ be a local geodesic in $T$. Then it corresponds to a chain of cells

$$
C_{0}^{\prime}, \ldots, C_{k}^{\prime}
$$

which correspond to the vertices in $q$, so that the intersections $C_{i}^{\prime} \cap C_{i+1}^{\prime}$ correspond to the edges in $q$. We will call the union

$$
C_{0}^{\prime} \cup \cdots \cup C_{k}^{\prime}
$$

the gallery $\operatorname{gal}(q)$ corresponding to the path $q$. We define a closed gallery as

$$
\bar{C}_{0} \cup \bar{C}_{1} \cup \cdots \cup \bar{C}_{k} .
$$

The number $k$ is the length of the gallery, it equals the length of the path $q$ in $T$. If $q$ is a geodesic in $T$, by abusing notation we will refer to $k$ as the distance between $C_{0}$ and $C_{k}$ and we will refer to the gallery $\operatorname{gal}(q)$ as a minimal gallery.

We call a segment $[x, y] \subset \bar{X}$ generic if the open segment $(x, y)$ is entirely contained in the union of open cells and open facets and is not contained in a facet. Therefore $(x, y)$ is not contained in a wall. Each generic segment $\sigma=[x, y]$ determines a path $q=p(\sigma)$ in $T$. The vertices $z_{0}, z_{1}, \ldots, z_{k}$ and edges of this path correspond to the open cells $C_{0}, \ldots, C_{k}$ and open facets in $X$ which cover the open segment $(x, y)$.

Lemma 4.11 For a generic segment $\sigma$, the path $p(\sigma)$ is a geodesic in $T$. 
Proof It follows immediately from convexity of the cells in $X$ and Part 1 of the Assumption 4, that $p(\sigma)$ is a local geodesic. Let $x, y$ denote the end-points of $p(\sigma)$. By Proposition 4.8, there exists a diagram $D_{\text {loc }} \subset Z$ bounded by a bigon $B$ with the vertices $x, y$, so that $p(\sigma)$ is contained in $D_{\text {loc }}^{(1)}$. If for each corridor $K \subset D_{\text {loc }}$, the intersection $p(\sigma) \cap K$ is a geodesic in $T$, then Proposition 4.8 implies that the path $p(\sigma)$ is a (global) geodesic.

Suppose that $p(\sigma)$ is not a global geodesic. Without loss of generality we may assume that $p(\sigma)$ contains no shorter non-geodesic subpath connecting vertices of $T$. Therefore $D$ is a corridor and the boundary of $D$ is formed by the union of $p(\sigma)$ and a geodesic $q$ which is shorter than $p(\sigma)$.

In this case there is a wall $H$ in $X$ whose intersection with the gallery $\operatorname{gal}(p(\sigma))$ is not connected. (The wall $H$ passes through the facets in $X$ corresponding to the interior edges of the corridor $D$.) However the segment $\sigma$ can intersect the wall $H$ transversally in at most one point. Contradiction.

Remark The above lemma is analogous to the familiar description of geodesics in Cayley graphs of Coxeter groups.

\subsection{Convexity of closed minimal galleries}

In this section we will prove Part 1 of Theorem 4.3. We will show that every two points $x, y$ in $\bar{X}$, the union of closed minimal galleries in $\bar{X}$ connecting $x$ and $y$ is convex. Given two cells $A, B$ in $X$, let $\operatorname{Gal}(A, B)$ and $\overline{\operatorname{Gal}}(A, B)$ denote the unions of all minimal galleries

$$
A^{\prime}=C_{0}^{\prime} \cup C_{1}^{\prime} \cup \cdots \cup C_{l}^{\prime}=B^{\prime},
$$

and minimal closed galleries

$$
\bar{A}=\bar{C}_{0} \cup \bar{C}_{1} \cup \cdots \cup \bar{C}_{l}=\bar{B},
$$

connecting $A$ to $B$. Since $\operatorname{Gal}(A, B)$ contains only finitely many cells (see Proposition 4.8), it follows that $\overline{\operatorname{Gal}}(A, B)$ is the closure of $\operatorname{Gal}(A, B)$ in $X$. The space $\overline{\operatorname{Gal}}(A, B)$ has a natural path metric induced by the pull-back of the Euclidean metric under the map dev: $\bar{X} \rightarrow \mathbb{R}^{n}$. With respect to this metric, $\overline{\operatorname{Gal}}(A, B)$ is a proper metric space, ie closed metric balls are compact.

We will prove existence of a segment in $\bar{X}$ connecting points $x, y$ by induction on the (combinatorial) distance between the cells containing $x$ and $y$.

In case when the points $x, y \in \bar{X}$ belong to the same cell, there is nothing to prove (since each cell is convex). 
Suppose that for every pair of cells $A, B$ in $X$ within distance $\leq k-1$, the union of closed galleries $\overline{\operatorname{Gal}}(A, B)$ is convex. Our goal is to prove that the same assertion holds for all cells within distance $k$.

Fix two cells $A, B$ which are distance $k$ apart and which correspond to vertices $a, b \in T$. Our main goal is to show that there exists a segment $[x, y] \subset \overline{\mathrm{Gal}}(A, B)$ for all $x \in \bar{A}, y \in \bar{B}$. Convexity of $\overline{\operatorname{Gal}}(A, B)$ will be proven as a corollary.

First, note that for each cell $D \subset \operatorname{Gal}(A, B)$ which is adjacent to $B$, the gallery $\operatorname{Gal}(A, D)$ is contained in $\operatorname{Gal}(A, B)$ and its projection to $T$ has diameter $k-1$. Thus $\overline{\mathrm{Gal}}(A, D)$ is convex by the induction hypothesis.

Let $\Phi:=\left\{F_{1}, \ldots, F_{l}\right\}$ denote the set of facets of $B$ which are contained in the interior of $\operatorname{Gal}(A, B)$. Then for each facet $F \in \Phi$ there exists a gallery

$$
A^{\prime}=C_{0}^{\prime} \cup C_{1}^{\prime} \cup \cdots \cup C_{k-1}^{\prime} \cup C_{k}^{\prime}=B^{\prime},
$$

so that $F=C_{k-1}^{\prime} \cap C_{k}^{\prime}$.

Fix a point $x$ in the open cell $A$ and let $y \in \bar{B}$ vary. Let $Y \subset \bar{B}$ denote the set of points $y \in \bar{B}$ such that there exists a segment $[x, y] \subset \overline{\operatorname{Gal}}(A, B)$. Let $Y_{\text {gen }}=Y_{\text {gen }}(x) \subset Y$ denote the set of points $y$ such that the segment $[x, y]$ is generic.

Let $Y_{\text {sing }} \subset Y$ denote the (possibly empty) closed set of points $y \in Y$ such that the segment $[x, y] \subset \overline{\mathrm{Gal}}(A, B)$ exists and passes through the set $\Lambda(F)$ for some codimension 2 face $F$ of $B$. (Recall that the set $\Lambda(F)$, which includes the origin, is defined as $\bar{F} \backslash F^{\prime}$.) Set $B_{\text {sing }}:=Y_{\text {sing }} \cap B$. Since $B$ has dimension $\geq 3$, it follows (from the dimension count and Part 3 of Assumption 3) that $B_{\text {sing }}$ does not locally separate $B$. Set

$$
B_{\text {reg }}:=B \backslash B_{\text {sing }}
$$

and

$$
Y_{\text {reg }}:=Y \cap B_{\text {reg }}
$$

Clearly,

$$
Y_{\text {gen }} \cap B \subset Y_{\text {reg }} \cap B \subset B_{\text {reg }} .
$$

In what follows, for a subset $W \subset B$ we let $\operatorname{int}_{\bar{B}}(W)$ denote the interior of $W$ in $\bar{B}$. For a subset $W \subset B_{\text {reg }}$ we let

$$
\operatorname{cl}_{B_{\text {reg }}}(W):=\operatorname{cl}(W) \cap B_{\text {reg }}
$$

denote the closure of $W$ in $B_{\text {reg }}$. 


\section{Proposition 4.12}

(1) $\operatorname{cl}\left(Y_{\text {gen }}\right) \subset Y$.

(2) $\operatorname{cl}_{B_{\text {reg }}}\left(Y_{\text {gen }} \cap B\right) \subset \operatorname{int}_{\bar{B}}\left(Y_{\text {reg }}\right)$.

(3) $\operatorname{int}_{\bar{B}}\left(Y_{\text {reg }}\right) \subset \operatorname{cl}_{B_{\text {reg }}}\left(Y_{\text {gen }} \cap B\right)$. Thus $\operatorname{cl}_{B_{\text {reg }}}\left(Y_{\text {gen }} \cap B\right)=\operatorname{int}_{\bar{B}}\left(Y_{\text {reg }}\right)$.

(4) Each facet $F \in \Phi$, is contained in $\operatorname{int}_{\bar{B}}\left(Y_{\text {reg }}\right)$.

\section{Proof}

(1) Consider a sequence $y_{j} \in Y_{\text {gen }}$ which converges to some $y \in \bar{B}$. Then, since $\overline{\mathrm{Gal}}(A, B)$ is closed and proper, the sequence of segments

$$
\left[x, y_{j}\right] \subset \overline{\operatorname{Gal}}(A, B)
$$

subconverges to a segment $[x, y] \subset \overline{\mathrm{Gal}}(A, B)$. Therefore $y \in Y$.

(2) For every $y \in Y_{\text {gen }}$ the segment $\sigma=[x, y] \subset \overline{\operatorname{Gal}}(A, B)$ crosses a facet $F$ of $B$ and an adjacent cell $D$. Since $\overline{\operatorname{Gal}}(A, D)$ is convex, it follows that there exists a (unique) point $z=z_{\sigma} \in[x, y]$ so that

$$
[z, y] \subset \bar{B}, \quad[x, z) \cap \bar{B}=\varnothing .
$$

Consider a sequence $y_{j} \in Y_{\text {gen }}$ which converges to a point $y \in B_{\text {reg. }}$. Then (similarly to the proof of 1 ), we can assume that the segments $\sigma_{j}=\left[x, y_{j}\right]$ converge to the segment $[x, y]$. Then the sequence $z_{j}:=z_{\sigma_{j}}$ converges to some $z \in \partial B \cap[x, y]$. Without loss of generality (by passing to a subsequence if necessary) we can assume that all $z_{j}$ 's belong to a common facet $F$ of $B$, so that $\bar{F}=\bar{D} \cap \bar{B}$, where $D \subset \operatorname{Gal}(A, B)$. Thus $z \in \bar{F} \subset \bar{D}$.

Since $y \in B_{\text {reg }}$, there are only three possibilities:

(a) $z \in F$.

(b) $z$ belongs to an (open) codimension 2 face contained in $\partial F$.

(c) $z$ does not belong to $X$; therefore it belongs to the intersection $\bar{D} \cap \bar{B}$ and is not contained in any closed cell other than $\bar{B}$ and $\bar{D}$ (see Part 2 of Assumption 3).

In all three cases, there exists a convex neighborhood $U$ of the point $z$ in

$$
\overline{\operatorname{Gal}}(A, D) \cup \bar{B}
$$

so that $U \cap X$ is also convex. (In case (a) it follows since $X$ is an affine manifold; in case (b) it follows from Part 1 of Assumption 4 and convexity of $\overline{\operatorname{Gal}}(A, D)$; in case (c) it follows from Part 2 of this assumption.) 
Recall that $x \in A \subset \operatorname{int}(\overline{\operatorname{Gal}}(A, D))$, the latter is convex. Thus, by Lemma 2.2,

$$
[x, z) \subset \operatorname{int}(\overline{\operatorname{Gal}}(A, D)) .
$$

According to Lemma 4.9, $\operatorname{int}(\overline{\operatorname{Gal}}(A, D)) \subset X$. Therefore $[x, z) \subset X$.

Convexity of $\bar{B}$ and Lemma 2.2 imply that $(z, y] \subset B \subset X$. Pick points $z^{\prime} \in \operatorname{int}(U) \cap[x, z), z^{\prime \prime} \in \operatorname{int}(U) \cap(z, y]$. Convexity of $\overline{\operatorname{Gal}}(A, B) \cap U$ then implies that $z \in \operatorname{int}(\operatorname{Gal}(A, B))$; hence the entire segment $[x, y)$ is contained in the interior of $\operatorname{Gal}(A, B)$. Therefore, since this interior is an affine manifold, there exists a neighborhood $V$ of $y$ in $B$ such that for each $y^{\prime} \in V$, there exists a segment $\left[x, y^{\prime}\right] \subset \operatorname{Gal}(A, B)$. In particular, every such $y^{\prime}$ belongs to $Y_{\text {reg }}$. Thus $y \in \operatorname{int}_{\bar{B}}\left(Y_{\text {reg }}\right)$.

(3) For $y \in \operatorname{int}_{\bar{B}}(Y)$, let $V \subset Y$ be an open ball containing $y$. Since the union of facets of the cell $C$ is dense in $C^{\prime} \backslash C$, we see that $V \backslash Y_{\text {gen }}$ is nowhere dense in $V$. Hence $Y_{\text {gen }} \cap V$ is dense in $V$ and therefore $y \in \operatorname{cl}\left(Y_{\text {gen }}\right)$. The assertion 3 follows.

(4) The proof of this assertion is analogous to the last part of the proof of 2. For each $y \in F$ the segment $[x, y]$ is contained in $\operatorname{int}(\operatorname{Gal}(A, B))$ and thus we can use a neighborhood $V$ of $y$ as above. To show that $y \in Y_{\text {reg }}$ note that the segment $[x, y] \subset X$ cannot pass through the origin. This segment is disjoint from the boundaries of codimension 2 faces of $B$ since the latter are contained in the boundary of $\overline{\operatorname{Gal}}(A, B)$.

Corollary 4.13 $\bar{B}=Y=\operatorname{cl}\left(Y_{\text {gen }}\right)$.

Proof By Part 3 of Proposition 4.12,

$$
\operatorname{cl}_{B_{\text {reg }}}\left(Y_{\text {gen }} \cap B\right)=\operatorname{int}_{\bar{B}}\left(Y_{\text {reg }}\right) .
$$

Thus $\operatorname{int}_{\bar{B}}\left(Y_{\text {reg }}\right)$ is both closed and open in $B_{\text {reg. }}$. By Part 4 of Proposition 4.12, the set $\operatorname{int}_{\bar{B}}\left(Y_{\text {reg }}\right)$ is nonempty. Since $B_{\text {reg }}$ is connected (recall that $B_{\text {sing }}$ does not locally separate $B$ ), we conclude that int ${ }_{\bar{B}}\left(Y_{\text {reg }}\right)=B_{\text {reg }}$. Thus $B_{\text {reg }} \subset Y$. Since $B_{\text {reg }}=B \backslash B_{\text {sing }}$ is dense is $\bar{B}$, by applying Part 1 of Proposition 4.12, we conclude that

$$
\bar{B} \subset \operatorname{cl}\left(B_{\text {reg }}\right)=\operatorname{cl}\left(\operatorname{int}_{\bar{B}}\left(Y \cap B_{\text {reg }}\right)\right)=\operatorname{cl}\left(Y_{\text {gen }}\right) \subset Y .
$$

Thus $\bar{B}=Y=\operatorname{cl}\left(Y_{\text {gen }}\right)$.

Therefore, for each point $y \in \bar{B}$ there exists a segment $[x, y] \subset \overline{\operatorname{Gal}}(A, B) \subset \bar{X}$. Recall that we assumed that $x \in A$, ie belongs to the open cell. For a point $y \in \bar{B}$ and $x \in \bar{A}$, pick a sequence $x_{j} \in A$ converging to $x$. Then there exist a sequence $y_{j} \in Y_{\text {gen }}\left(x_{j}\right)$ 
which converges to $y$; the segments $\left[x_{j}, y_{j}\right]$ are all contained in $\overline{\operatorname{Gal}}(A, B)$. Therefore, by properness of $\overline{\operatorname{Gal}}(A, B)$, we conclude that the segments $\left[x_{j}, y_{j}\right]$ converge to a segment $[x, y] \subset \overline{\operatorname{Gal}}(A, B)$.

Thus we proved that for each pair of cells $A$ and $B$ within distance $\leq k$, and each pair of points $x \in \bar{A}, y \in \bar{B}$, there exists a segment $[x, y] \subset \overline{\mathrm{Gal}}(A, B) \subset \bar{X}$.

Claim 4.14 The set $\overline{\operatorname{Gal}}(A, B)$ is convex for every pair of cells $A, B$ which are distance $k$ apart.

Proof Pick two cells $A, B$ which are distance $k$ apart and which correspond to vertices $a, b \in T$. Recall that the union of galleries $\overline{\operatorname{Gal}}(A, B)$ projects to the convex subset geo $(a, b)$ of $T$. Let $z, w \in \overline{\operatorname{Gal}}(A, B)$. Then these points belong to cells $\bar{C}, \bar{D} \subset$ $\overline{\operatorname{Gal}}(A, B)$. These cells project to vertices $c, d \in \operatorname{geo}(a, b)$. Since geo $(a, b)$ is convex, the union of closed galleries $\overline{\operatorname{Gal}}(C, D)$ projects to a subset $\operatorname{geo}(c, d) \subset \operatorname{geo}(a, b)$. Moreover, since $\operatorname{diam}(\operatorname{geo}(a, b))=k$, it follows that $\operatorname{diam}(\operatorname{geo}(c, d)) \leq k$ and thus $d(C, D) \leq k$. Hence $[z, w] \subset \overline{\operatorname{Gal}}(C, D)$ and $\overline{\operatorname{Gal}}(C, D)$ is contained in $\overline{\operatorname{Gal}}(A, B)$. Therefore $[z, w] \subset \overline{\operatorname{Gal}}(A, B)$ and convexity of $\overline{\operatorname{Gal}}(A, B)$ follows.

Hence, by induction, we conclude that $\bar{X}$ is convex. It follows that dev: $\bar{X} \rightarrow \mathbb{R}^{n}$ is a continuous map onto a convex cone $K \subset \mathbb{R}^{n}$. To see that this map is injective note that for distinct points $x, y \in \bar{X}$ there exists a segment $[x, y] \subset X$; since the restriction of $\operatorname{dev}$ to $[x, y]$ is injective, it follows that $\operatorname{dev}(x) \neq \operatorname{dev}(y)$. Thus $\operatorname{dev}: \bar{X} \rightarrow K$ is a continuous bijection. (At the moment we do not know is this map is a homeomorphism since $\bar{X}$ is not locally compact.)

This proves the first assertion of Theorem 4.3.

\subsection{Convexity of $X$}

We now prove convexity of $X$. The idea is to use Lemma 4.9 in conjunction with Lemma 2.2. Let $x, y \in X$. We first give a proof in the easier case when $x$ belongs to an open cell $A$. Let $\bar{B}$ denote a closed cell containing $y$. Then the segment $[x, y] \subset \bar{X}$ is contained in the closed gallery $W=\overline{\operatorname{Gal}}(A, B)$. As $\operatorname{dev}(W)$ is a closed convex subset of $\mathbb{R}^{n}$ and $\operatorname{dev}(x)$ belongs to the interior of $\operatorname{dev}(W)$, by Lemma 2.2 , we conclude that

$$
\operatorname{dev}([x, y]) \subset \operatorname{int}(\operatorname{dev}(W)) \cup\{\operatorname{dev}(y)\} .
$$

Since $\operatorname{dev} \mid W$ is a homeomorphism to its image, the open segment $(x, y)$ is contained in the interior of $W$. Thus, by Lemma 4.9, this segment is entirely contained in $X$. As $x, y$ belong to $X$, it follows that $[x, y] \subset X$. 
Consider now the general case. Our proof will be similar to the above argument, except that we will have to generalize slightly the proof of Lemma 2.2. Pick a relatively compact neighborhood $U$ of $x$ in $X$. Then $U$ is covered by finitely many cells $A_{j}^{\prime} \subset X$. Choose a cell $B^{\prime}$ containing $y$. For every $x^{\prime} \in U$, the segment $\left[x^{\prime}, y\right] \subset \bar{X}$ is covered by a finite union of closed faces contained in

$$
W=\bigcup_{j} \overline{\operatorname{Gal}}\left(A_{j}, B\right) .
$$

Therefore the cone $\operatorname{Cone}_{y}(U)$ with the tip $y$ and the base $U$ is covered by finitely many closed cells. Thus the developing map dev sends $\operatorname{Cone}_{y}(U)$ homeomorphically onto a convex subset

$$
\Sigma:=\operatorname{dev}\left(\operatorname{Cone}_{y}(U)\right)=\operatorname{Cone}_{\operatorname{dev}(y)}(\operatorname{dev}(U)) \subset \mathbb{R}^{n} .
$$

Clearly, the open segment $(\operatorname{dev}(x), \operatorname{dev}(y))$ is contained in the interior of $\Sigma$. It follows that the open segment $(x, y)$ is also contained in the interior of $W$. Hence, according to Lemma 4.9, the open segment $(x, y)$ is contained in $X$.

Thus dev is a continuous bijection of $X$ onto a convex homogeneous cone $K^{0}$ in $\mathbb{R}^{n}$. Invariance of domain implies that dev: $X \rightarrow K^{0}$ is a homeomorphism. Since $0 \in \bar{X} \backslash X$ and dev: $\bar{X} \rightarrow \mathbb{R}^{n}$ is $1-1$, it follows that $0 \notin K^{0}$ and hence $K^{0}$ is a proper cone.

\section{Products of matrices}

In this section we will consider the following problem.

Problem 6 Let $G$ be a Lie group with a fixed collection of 1-parameter subgroups $G_{1}, \ldots, G_{k} \subset G$. Analyze the image of the map

$$
\text { Prod: } \prod_{i=1}^{k} G_{i} \rightarrow G
$$

given by $\operatorname{Prod}\left(g_{1}, \ldots, g_{k}\right)=g_{1} \bullet \cdots \bullet g_{k}$.

In the case when $G=S O(3)$, this problem is ultimately related to the variety of geodesic $k$-gons in $S^{3}$ with the fixed side-lengths, Kapovich and Millson [13]. (See Kapovich and Millson [12] or Maubon [15], for the relation of this product problem to bending deformations of flat conformal structures.) 
Here we consider the case of $G=G L(2, \mathbb{R})$; the subgroups $G_{i}$ are orthogonal conjugates of the group of diagonal matrices $\left\{\operatorname{Diag}\left(1, e^{t}\right), t \in \mathbb{R}\right\}$. The specific problem which we are interested in is as follows.

Problem 7 Describe the intersection of the image of Prod with the orthogonal subgroup $S O(2) \subset G L(2, \mathbb{R})$.

Let $\mathfrak{g l}(2, \mathbb{R})=\mathfrak{p} \oplus \mathfrak{o}(2)$ denote the Cartan decomposition of the Lie algebra of $G L(2, \mathbb{R})$. The Lie algebras $\mathfrak{p}_{i}$ of $G_{i}$ 's are contained in $\mathfrak{p}$. Let $e:=(1, \ldots, 1) \in \prod_{i} G_{i}$. Then derivative

$$
d \operatorname{Prod}_{e}: \oplus_{i} \mathfrak{p}_{i} \rightarrow \mathfrak{g l}(2, \mathbb{R})
$$

is the map

$$
\left(\xi_{1}, \ldots, \xi_{k}\right) \mapsto \sum_{i} \xi_{i}
$$

Therefore its image is contained in $\mathfrak{p}$ and hence is orthogonal to $\mathfrak{o}(2)$. Thus one cannot approach Problem 7 by making infinitesimal calculations.

There is probably a purely algebraic or analytic solution to Problem 7; we will use hyperbolic geometry instead.

The main result of this section is Theorem 5.3, which shows that for certain 1-parameter subgroups $G_{i}$ of $G L(2, \mathbb{R})(i=1, \ldots, 4)$, the image of the product map

$$
\text { Prod: } G_{1} \times \cdots \times G_{4} \rightarrow G L(2, \mathbb{R})
$$

contains rotations by all the angles $\tau \in(-\pi / 4,0]$.

We start by describing the subgroups $G_{i}$. Given a basis $(v, w)$ of $\mathbb{R}^{2}$ and $t \in \mathbb{R}$ we define the matrix

$$
A=A_{v, w, t}
$$

to be the linear transformation which fixes $v$ and sends $w$ to $e^{t} w$ :

$$
A=\left[\begin{array}{ll}
1 & 0 \\
0 & e^{t}
\end{array}\right] \text {. }
$$

The 1-parameter group $G_{1}$ will consist of the transformations $A_{v, w, t}$, where $\{v, w\}$ is an orthonormal basis in $\mathbb{R}^{2}$. The subgroups $G_{2}, G_{3}, G_{4}$ will be conjugate to $G_{1}$ by the rotations by the angles $\pi / 4, \pi / 2$ and $3 \pi / 4$.

Our strategy for analyzing the product map Prod is to understand how the groups $G_{i}$ act on the hyperbolic plane.

Consider the projective action of $G L(2, \mathbb{R})$ on the circle $\mathbb{R} P^{1}$ (which we identify with the boundary of the hyperbolic plane $\left.\mathbb{\boxplus}^{2}\right)$. We will use the notation $[A] \in P G L(2, \mathbb{R})$ 
for the projection of the matrix $A \in G L(2, \mathbb{R})$. The vectors $v, w$ project to the fixed points $[v],[w]$ of the projective transformation $[A]$. Then $[v]$ is the repulsive and $[w]$ is the attractive fixed point of $[A]: \mathbb{R} P^{1} \rightarrow \mathbb{R} P^{1}$, provided that $t>0$. If $t<0$, then the attractive and repulsive fixed points are interchanged.

We identify the hyperbolic plane $\mathbb{H}^{2}$ with the unit disk in $\mathbb{R}^{2}$ in such a way that the group $O(2) \subset G L(2, \mathbb{R})$ fixes the origin 0 in $\mathbb{H}^{2}$. The projective line $\mathbb{R} P^{1}$ becomes the ideal boundary of $\mathbb{\boxplus}^{2}$.

Then the hyperbolic geodesic $L_{A}=\overline{[v][w]} \subset \mathbb{M}^{2}$ invariant under $[A]$ passes through the origin 0 (and hence is a Euclidean straight line). We parameterize the geodesic $L_{A}$ with the unit speed and orient $L_{A}$ in the direction from $[v]$ to $[w]$, thereby identifying it with the real line. The origin in $\mathbb{U}^{2}$ corresponds to zero in $\mathbb{R}$. We let $L_{A}^{ \pm}$denote the positive and negative rays (starting at 0 ) in $L_{A}$ corresponding to this orientation.

In these coordinates, the isometry $[A]$ acts on $L_{A}$ by $r \mapsto r+t$. The isometry $\left[A_{w, v, t}\right]$ acts on $\mathbb{H}^{2}$ by the translation $r \mapsto r-t$ along the geodesic $L_{A}$. By considering the action of $S O(2)$ by conjugation we see the following.

Let $R=R_{\phi} \in S O(2)$ be the rotation by the angle $\phi$. Then the matrix

$$
R_{\phi} A_{v, w, t} R_{\phi}^{-1}=A_{R(v), R(w), t}
$$

acts on $\mathbb{T}^{2}$ by the translation $r \mapsto r+t$ along the geodesic

$$
\overline{R_{\phi / 2}([v]) R_{\phi / 2}([w])} \text {. }
$$

We now consider the 1-parameter groups

$$
\begin{array}{llrl}
G_{1} & =\left\{A_{e_{1}, e_{2}, t}: t \in \mathbb{R}\right\}, & & G_{2}=R_{\pi / 4} G_{1} R_{-\pi / 4}, \\
G_{3} & =R_{\pi / 2} G_{1} R_{-\pi / 2}=\left\{A_{e_{2}, e_{1}, t}: t \in \mathbb{R}\right\}, & & G_{4}=R_{-\pi / 4} G_{1} R_{\pi / 4} .
\end{array}
$$

Geometrically, these are groups of translations along two orthogonal hyperbolic geodesics $L_{1}$ and $L_{2}$ in $\mathbb{R}^{2}\left(G_{i}\right.$ and $G_{i+2}$ translate along $L_{i}$ in the opposite directions, $i=1,2)$. Given a matrix $A_{i} \in G_{i}$ we let $\ell_{i}:=\ell\left(A_{i}\right)$ denote the translation length of $\left[A_{i}\right]$ along its invariant geodesic; here we are ignoring the orientation so that $\ell_{i} \geq 0$.

Thus, in order for $A_{i} \in G_{i}, i=1, \ldots, 4$ to have the product equal to $R_{\phi}$, it is necessary and sufficient to have:

(1) The product of the eigenvalues of $A_{i}$ 's is equal to 1 (ie the product of four matrices is in $S L(2, \mathbb{R})$ ). Equivalently,

$$
t_{1}+t_{2}+t_{3}+t_{4}=0 .
$$


(2) The composition of the hyperbolic translations

$$
\left[A_{1}\right] \circ\left[A_{2}\right] \circ\left[A_{3}\right] \circ\left[A_{4}\right]
$$

is the rotation $R_{\phi / 2}$ around the origin in $\mathbb{H}^{2}$. In particular, the above product of hyperbolic isometries has to fix the intersection $L_{1} \cap L_{2}$.

Remark Similar description, of course, will be valid for more general choices of 1 -parameter groups $G_{i}$ which are conjugate to $G_{1}$ by rotations $R_{\theta_{i}}, i=1, \ldots, k$.

With this geometric interpretation it is clear, for instance, that the product $A_{1} A_{2} A_{3}$ is never a nontrivial rotation. The reason is that unless $A_{2}=1,\left[A_{1}\right]=\left[A_{3}\right]^{-1}$, the product of the hyperbolic isometries does not fix the origin.

We now make the situation a bit more symmetric and require that

$$
\ell_{1}=\ell_{4}, \quad \ell_{2}=\ell_{3} .
$$

In particular, the Condition 1 will be satisfied provided that

$$
t_{1}, t_{3}>0, \quad t_{2}, t_{4}<0 .
$$

We then consider the images of the origin under the compositions of the isometries $\left[A_{1}\right],\left[A_{2}\right],\left[A_{3}\right],\left[A_{4}\right]$. We let $x_{0}:=x_{4}:=0 ; x_{i}:=\left[A_{i}\right]\left(x_{i+1}\right), i=1, \ldots, 4$. Given a number $\theta \in\left(0, \frac{\pi}{2}\right)$ set

$$
\alpha=\alpha(\theta):=\frac{\pi}{2}-\theta \text {. }
$$

Lemma 5.1 For every $\theta \in\left[0, \frac{\pi}{2}\right)$ there exists a pair of continuous functions $\ell_{i}=$ $\ell_{i}(\theta), i=1,2$, so that:

(1)

$$
\left\{\begin{array}{c}
\cosh \left(\ell_{2}\right)=\cosh \left(\ell_{1}\right) \sin (\alpha) \\
\sinh ^{2}\left(\ell_{1}\right)=\cos (\alpha)
\end{array}\right.
$$

In particular, $\ell_{i}(0)=0, i=1,2$.

(2) For $\ell_{i}=\ell_{i}(\theta)$ the composition

$$
\left[A_{1}\right] \circ\left[A_{2}\right] \circ\left[A_{3}\right] \circ\left[A_{4}\right]
$$

is the (counter-clockwise) rotation $R_{\theta}$ around the origin $0 \in \mathbb{M}^{2}$ by the angle $\theta$. 
Proof Let $L_{1}, L_{2}$ be the pair of oriented geodesics in $\mathbb{\boxplus}^{2}$ (invariant under the subgroups $\left[G_{1}\right]=\left[G_{3}\right],\left[G_{2}\right]=\left[G_{4}\right]$ respectively) which intersect orthogonally at the origin.

We orient the geodesics $L_{1}, L_{2}$ away from the points $\left[e_{1}\right],\left[R_{\pi / 4}\left(e_{1}\right)\right]$ fixed by $\left[A_{1}\right] \in$ $\left[G_{1}\right],\left[A_{2}\right] \in\left[G_{2}\right]$. Let $L_{i}^{+}$denote the positive half-rays in these geodesics.

Recall that $\alpha \in\left(0, \frac{\pi}{2}\right]$ and that we will be using the translation parameters so that

$$
t_{1}, t_{3}>0, \quad t_{2}, t_{4}<0 .
$$

The key observation is that for each $\alpha \in\left(0, \frac{\pi}{2}\right]$ there exists a unique geodesic quadrilateral (a Lambert quadrilateral) $Q_{\alpha}=\left[0, y_{1}, x_{2}, y_{2}\right]$ in $\mathbb{W}^{2}$ with the three right angles (at the vertices $0, y_{1} \in L_{1}^{+}, y_{2} \in L_{2}^{+}$) and the angle $\alpha$ at the vertex $x_{2}$. See Figure 8. The orientation on $Q_{\alpha}$ given by the cyclic ordering of its vertices $x_{0}, \ldots, x_{3}$, is clockwise, which corresponds to the assumption that $\theta \geq 0$.

Set

$$
\ell_{2}=\ell_{3}:=d\left(0, y_{1}\right)=d\left(0, y_{2}\right)
$$

and

$$
\ell_{1}=\ell_{4}:=d\left(y_{2}, x_{2}\right)=d\left(y_{1}, x_{2}\right) .
$$

It is clear that $\ell_{1}, \ell_{2}$ are continuous functions of $\theta$ so that $\ell_{i}(0)=0$. The equations relating $\alpha, \ell_{1}, \ell_{2}$ follow immediately from the hyperbolic trigonometry, see Beardon [1, Theorem 7.17.1].

Choose points $x_{1} \in L_{1}^{+}, x_{3} \in L_{2}^{+}$so that

$$
d\left(0, x_{1}\right)=\ell_{1}=d\left(0, x_{3}\right)=\ell_{4} .
$$

Now take the hyperbolic translations $g_{1}, g_{3}$ along $L_{1}$ sending $x_{1}$ to 0 and 0 to $y_{1}$, respectively. Define the hyperbolic translations $g_{2}, g_{4}$ along $L_{2}$ sending $y_{2}$ to 0 and 0 to $x_{3}$, respectively. Thus the isometries $g_{1}, g_{4}$ have the translation lengths $\ell_{1}=\ell_{4}$; the isometries $g_{2}, g_{3}$ have the translation lengths $\ell_{2}=\ell_{3}$.

It is clear from the Figure 8 that

$$
g_{2}\left(x_{2}\right)=x_{1}, g_{3}\left(x_{3}\right)=x_{2}
$$

and therefore

$$
g_{1} \circ g_{2} \circ g_{3} \circ g_{4}(0)=0 \text {. }
$$

Hence the above composition of translations is a certain rotation $R_{\phi}$ around the origin. In order to compute the angle $\phi$ of rotation take two vectors $\xi_{1}, \xi_{2} \in T_{0} \mathbb{M}^{2}$ tangent 
to the geodesic rays $L_{1}^{-}$and $L_{2}^{-}$, respectively. Then the images of $\xi_{1}, \xi_{2}$ under the derivatives

$$
d\left(\left(g_{1} \circ g_{2}\right)^{-1}\right), \quad d\left(g_{4} \circ g_{3}\right)
$$

are tangent to the geodesic segments $\overline{x_{2} y_{2}}, \overline{x_{2} y_{1}}$ respectively. Therefore the angle $\phi$ equals $\frac{\pi}{2}-\alpha$ (the rotation is in the counter-clockwise direction). Thus $\phi=\theta$.

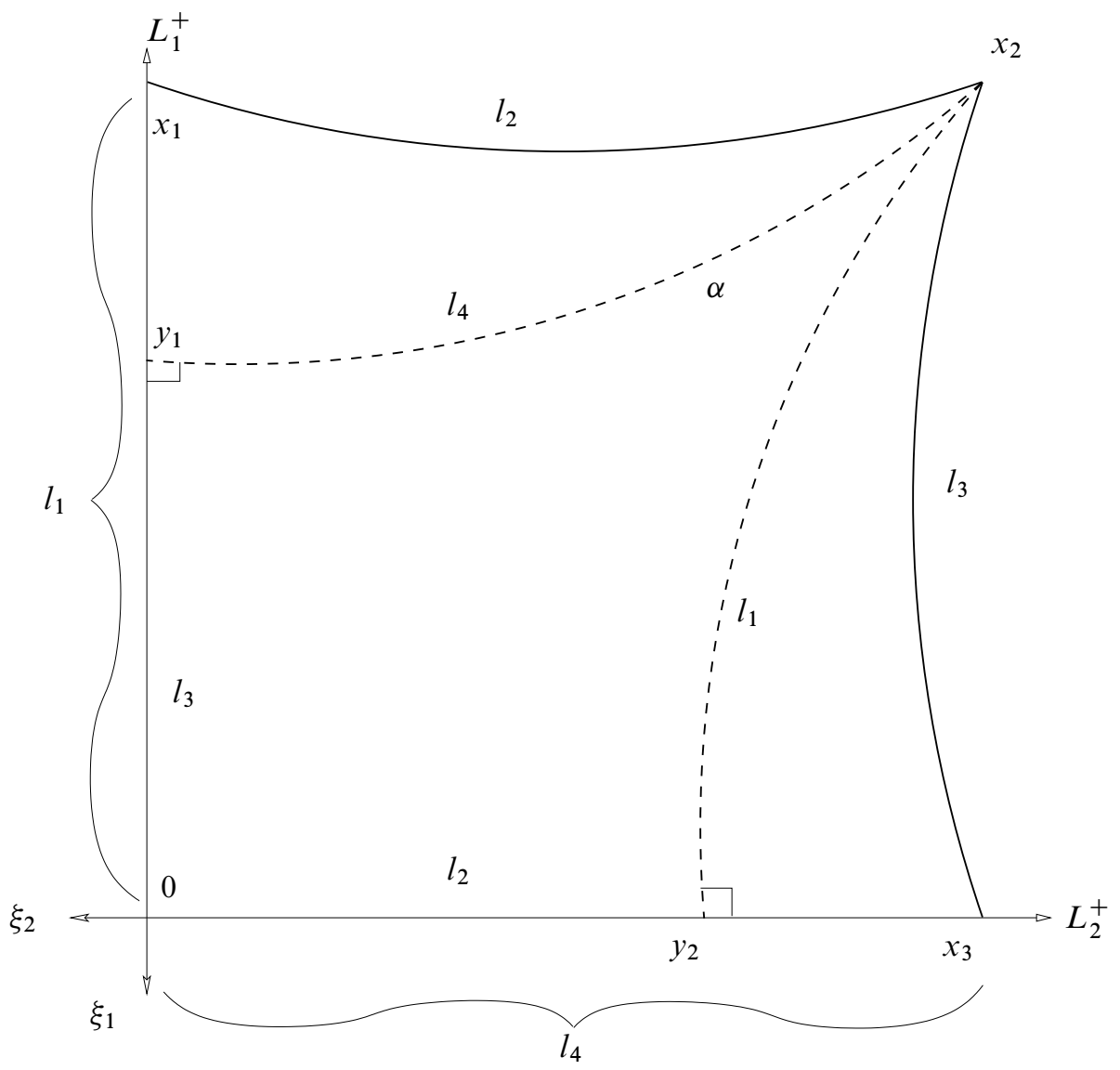

Figure 8: Lambert quadrilateral.

Let $A_{i} \in G_{i}$ denote the matrices corresponding to the hyperbolic translations $g_{i}$.

\section{Corollary 5.2}

$$
A_{1} \cdot A_{2} \cdot A_{3} \cdot A_{4}=R \frac{\alpha}{2}-\frac{\pi}{4} .
$$

Therefore we get the following theorem. 
Theorem 5.3 For each $\tau \in\left(-\frac{\pi}{4}, 0\right]$ there is a unique pair of numbers $t_{1} \geq 0, t_{2} \leq 0$ so that for the set of parameters

$$
\vec{t}=\left(t_{1}, t_{2},-t_{2},-t_{1}\right)
$$

the product of the corresponding matrices equals the rotation $R_{\tau}$. Moreover, the function $\vec{t}$ depends continuously on $\tau$.

Projective generalization Let $P \subset \mathbb{R} P^{n}$ be a projective hyperplane, $p \in \mathbb{R} P^{n} \backslash P$ and $t \in \mathbb{R}$. Then there exists a unique map $A=A_{P, p, t} \in P G L(n+1, \mathbb{R})$ satisfying:

(1) $A$ fixes $P \cup\{p\}$ pointwise.

(2) The derivative $d A_{p}$ equals $e^{t} I$.

If we identify $\mathbb{R} P^{n}$ with the compactification of the affine space $\mathbb{A}^{n}$, so that $p=$ $[\infty, 0, \ldots, 0] \in \mathbb{R} P^{n} \backslash \mathbb{A}^{n}$ and $P \cap \mathbb{A}^{n}$ is the affine hyperplane

$$
\left\{\left(x_{1}, \ldots, x_{n}\right): x_{2}=\cdots=x_{n}=0\right\}
$$

passing through the origin 0 , then

$$
A=\left[\begin{array}{cccc}
e^{t} & 0 & \ldots & 0 \\
0 & 1 & \ldots & 0 \\
\ldots & \ldots & \ldots & \ldots \\
0 & 0 & \ldots & 1
\end{array}\right]
$$

Suppose now that $n=2, \mathbb{R}^{2}$ is the affine patch of $\mathbb{R} P^{2}$. Let $P \subset \mathbb{R} P^{2}$ be the projective line tangent to the unit vector $v \in T_{0} \mathbb{R}^{2}, p \in \mathbb{R} P^{2} \backslash \mathbb{R}^{2}$ be the point at infinity so that the corresponding line $l$ through the origin contains the unit vector $w$ orthogonal to $v$. Then

$$
A_{P, p, t}=A_{v, w, t} \in G L(2, \mathbb{R}) \subset P G L(3, \mathbb{R}) .
$$

The identity extension of this linear transformation to the element $\hat{A} \in G L(n, \mathbb{R}) \subset$ $P G L(n+1, \mathbb{R})$ equals

$$
A_{Q, q, t}
$$

where $Q$ is the projective hyperplane through the origin orthogonal to $w$, the point $q \in \mathbb{R} P^{n} \backslash \mathbb{R}^{n}$ corresponds to the line $l$ as above.

Consider now a collection $P_{1}, P_{2}, P_{3}, P_{4}$ of projective hyperplanes in $\mathbb{R} P^{n}$ passing through the origin, so that the intersection

$$
\bigcap_{i} P_{i}=S
$$


is a codimension 2 projective subspace in $\mathbb{R} P^{n}$. We assume that the consecutive hyperplanes intersect at the angles $\frac{\pi}{4}$. For each $P_{i}$ let $p_{i} \in \mathbb{R} P^{n} \backslash \mathbb{R}^{n}$ be the "dual point" ie the corresponding line $l_{i}$ through the origin is orthogonal to $P_{i}$.

Remark Somewhat more invariantly, one can describe this setting as follows. We fix a positive definite bilinear form on $\mathbb{R} P^{n}$ so that the points $p_{i}$ are dual to the hyperplanes $P_{i}$. Therefore the assumption that

$$
\bigcap_{i} P_{i}=S
$$

is a codimension 2 projective hyperplane in $\mathbb{R} P^{n}$ implies that $\left\{p_{1}, \ldots, p_{4}\right\}$ is contained in a projective line $s \subset \mathbb{R} P^{n}$ dual to $S$. We then are assuming that the points $p_{1}, \ldots, p_{4}$ are cyclically ordered on $s$ so that the distance between the consecutive points is $\pi / 4$. (Note that $\mathbb{R} P^{1}=s$ has length $\pi$.)

Then Theorem 5.3 can be restated as follows.

Theorem 5.4 For each angle $\tau \in\left(-\frac{\pi}{4}, 0\right]$ there is a unique set of parameters $\vec{t}=$ $\left(t_{1}, t_{2}, t_{3}, t_{4}\right)=\left(t_{1}, t_{2},-t_{2},-t_{1}\right)$ with $t_{1} \geq 0, t_{2} \leq 0$ so that the composition of the corresponding projective transformations

$$
A_{P_{1}, p_{1}, t_{1}} \circ \cdots \circ A_{P_{4}, p_{4}, t_{4}}
$$

equals the rotation $R_{\tau}$ around $S$ by the angle $\tau$, fixing $S$ pointwise. Moreover, the function $\vec{t}$ depends continuously on $\tau$.

\section{Bending}

In this section we review the bending deformation of projective structures. Recall that in the end of the previous section we defined projective transformations

$$
A=A_{P, p, t} \in P G L(n+1, \mathbb{R})
$$

corresponding to the triples $(P, p, t)$, where $P \subset \mathbb{R} P^{n}$ is a projective hyperplane, $p \in \mathbb{R} P^{n} \backslash P$ and $t \in \mathbb{R}$.

Before proceeding with the general definition we start with a basic example of bending. Let $B$ denote the open unit ball in $\mathbb{R}^{n}$, which we will identify with the hyperbolic $n$-space. Let $H_{1}, \ldots, H_{k}$ denote disjoint hyperbolic hyperplanes in $B$ so that $H_{i}$ separates $H_{i-1}$ from $H_{i+1}, i=2, \ldots, k-1$. We assume that the $H_{i}$ are cooriented 


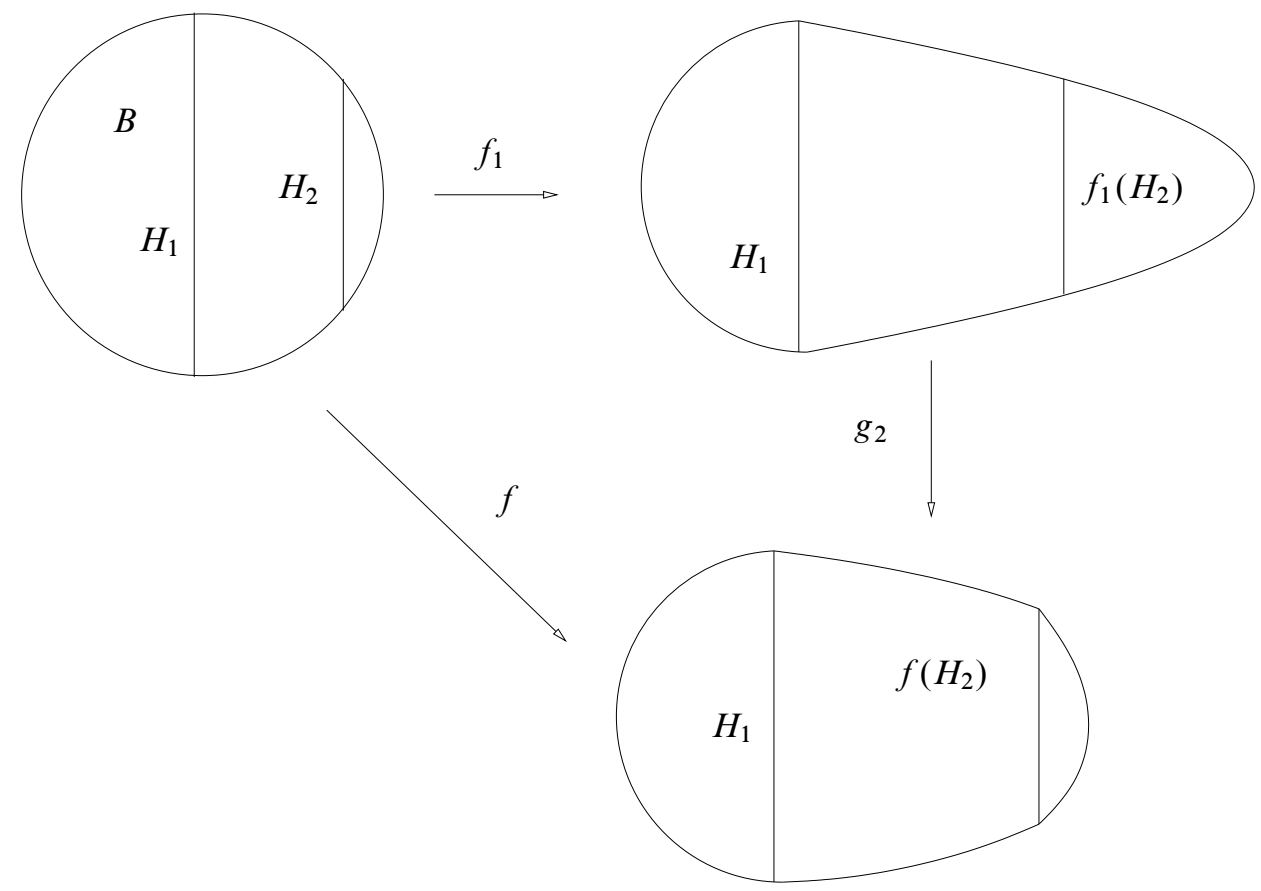

Figure 9: Projective bending.

in such a way that $H_{i+1}$ is to the right from $H_{i}, i=1, \ldots, k$. Let $H_{i}^{ \pm}$denote the half-space in $B$ bounded by $H_{i}$ and lying to the left (resp. right) from $H_{i}$. We set

$$
B_{i}:=H_{i}^{+} \cap H_{i+1}^{-}, \quad i=1, \ldots, k-1
$$

and

$$
B_{0}:=H_{1}^{-}, B_{k}:=H_{k}^{+} .
$$

Let $P_{i}=\operatorname{Span}\left(H_{i}\right)$ denote the projective hyperplane containing $H_{i}$ and let $p_{i} \in \mathbb{R} P^{n}$ denote the point dual to $P_{i}$ with respect to the quadratic form where $B$ is the unit ball. Choose real numbers $t_{1}, \ldots, t_{k}$. Our goal is to bend $B$ projectively in $\mathbb{R} P^{n}$ along the hypersurfaces $H_{i}$ with the bending parameters $t_{i}, i=1, \ldots, k$. Let $A_{i}:=A_{P_{i}, p_{i}, t_{i}}$, $i=1, \ldots, k$.

We will do bending inductively. First, let $f_{1}: B \rightarrow \mathbb{R} P^{n}$ denote the map which is the identity on $H_{1}^{-}$and $A_{1}$ on $H_{1}^{+}$. We then would like to bend $B_{1}:=f_{1}(B)$ along $f_{1}\left(H_{2}\right)$. The corresponding bending map $g_{2}$ is the identity on $f_{1}\left(H_{2}^{-}\right)$and $A_{2}^{\prime}$ on $f_{1}\left(H_{2}^{+}\right)$, where

$$
A_{2}^{\prime}=A_{1} \circ A_{2} \circ A_{1}^{-1}
$$


Therefore the map

$$
f_{2}: B \rightarrow g_{2}\left(B_{1}\right)
$$

equals to Id on $B_{0}=H_{1}^{-}$, to $A_{1}$ on $B_{1}=H_{1}^{+} \cap H_{2}^{-}$and to $A_{1} \circ A_{2}$ on $H_{2}^{+}$. Continuing in the fashion inductively we eventually obtain the bending map

$$
f: B \rightarrow \mathbb{R} P^{n}
$$

so that the restriction $f \mid B_{i}$ equals

$$
A_{1} \circ \cdots \circ A_{i}, i=1, \ldots, k-1,
$$

and $f \mid B_{0}=$ Id. The same construction works for an arbitrary locally finite collection $\mathcal{H}$ of disjoint hyperplanes $H_{i}$. We then pick a component $C_{0}$ of $Y=B \backslash \cup_{i} H_{i}$ where the bending map $f$ is the identity. Given a component $C_{k}$ of $Y$ we take the finite subcollection $\left\{H_{1}, \ldots, H_{k}\right\}$ of hyperplanes in $\mathcal{H}$ separating $C_{0}$ from $C_{k}$. We then repeat the above construction of bending map to define the restriction of bending to $C_{k}$.

Remark More generally, if $(L, \mu)$ is a measured codimension 1 totally-geodesic lamination in $B$, we can define projective bending with respect to this lamination. However, in view of Ratner's theorem, this generalization is not useful in the context of bendings of compact manifolds of dimension $\geq 3$.

We now give the general definition of bending.

Let $M$ be a projective $n$-manifold (or, more generally, an orbifold). Let $f: \tilde{M} \rightarrow \mathbb{R} P^{n}$ and $\rho: \Gamma=\pi_{1}(M) \rightarrow P G L(n+1, \mathbb{R})$ be the developing map and the holonomy of $M$.

Let $L \subset M$ be a proper hypersurface (possibly contained in the boundary of $M$ ); let $\widetilde{L} \subset \tilde{M}$ be the preimage of $L$ in the universal cover of $M$.

We call the hypersurface $L$ flat if it satisfies the following:

(1) Each point $x \in L$ has a neighborhood $U \subset L$ so that the developing map sends $U$ to an open subset of a projective hyperplane in $\mathbb{R} P^{n}$.

(2) For a component $\tilde{L}_{i} \subset \tilde{L}$ let $\Gamma_{i}$ be the stabilizer of $\tilde{L}_{i}$ in $\Gamma$. The group $\rho\left(\Gamma_{i}\right)$ stabilizes the projective hyperplane $P_{i}=\operatorname{Span}\left(f\left(\tilde{L}_{i}\right)\right) \subset \mathbb{R} P^{n}$.

We then require that for each $\widetilde{L}_{i}$, the group $\rho\left(\Gamma_{i}\right)$ has an isolated fixed point $p_{i} \in \mathbb{R} P^{n}$ which is disjoint from $P_{i}$. 
We define a cooriented weighted hypersurface $L$ in $M$ as follows. Consider the union $L$ of flat connected cooriented hypersurfaces $L_{i}$ in $M$, which intersect the boundary of $M$ transversally and so that for distinct $L_{i}, L_{j}$

$$
L_{i} \cap L_{j} \cap \operatorname{int}(M)=\varnothing .
$$

In this paper we will be assuming that the collection of hypersurfaces $L_{i}$ is locally finite in $M$, although one can make the discussion more general.

Definition 6.1 A weight for $L$ is a locally constant function $\mu: \operatorname{L\cap } \operatorname{int}(M) \rightarrow(0, \infty)$, $\mu: L_{i} \mapsto e^{t_{i}}$.

A weighted cooriented hypersurface is the pair $\lambda=(L, \mu)$. The weighted hypersurface $\lambda=(L, \mu)$ lifts to a cooriented weighted hypersurface $\tilde{\lambda}=(\tilde{L}, \tilde{\mu})$ in $\tilde{M}$.

We now define the bending deformation $c_{\lambda}$ of the projective structure $c$ on $M$ along the weighted hypersurface $\lambda$. The structure $c_{\lambda}$ will have the developing map $\operatorname{dev}_{\lambda}=f_{\lambda}$ satisfying the following properties.

For each component $H=\widetilde{L}_{i}$ of $\widetilde{L}$ with the stabilizer $\Gamma_{i}$, let $P_{i}, p_{i}$ denote the projective hyperplane and a point in $\mathbb{R} P^{n}$ stabilized by $\rho\left(\Gamma_{i}\right)$ as above. Let $H_{-}, H_{+}$ denote the components of $\tilde{M} \backslash \widetilde{L}$ to the left and to the right of $H$ (with respect to the coorientation). We then require that there exists a projective transformation $g \in P G L(n+1, \mathbb{R})$ so that

$$
\begin{aligned}
& f_{\lambda}\left|H_{-}=g \circ f\right| H_{-}, \\
& f_{\lambda}\left|H_{+}=A_{P, p, t} \circ g \circ f\right| H_{+} .
\end{aligned}
$$

The map $f_{\lambda}$ with these properties exists and can be constructed inductively similarly to our discussion of bending of the hyperbolic space. It is clear that $f_{\lambda}$ is unique up to postcomposition with projective transformations of $\mathbb{R} P^{n}$. The description of $f_{\lambda}$ implies that this map is a local homeomorphism. Since $\tilde{\lambda}$ is $\Gamma$-invariant, it follows that the map $f_{\lambda}$ is equivariant with respect to a homomorphism $\rho_{\lambda}: \Gamma \rightarrow P G L(n+1, \mathbb{R})$.

Thus the pair $\left(f_{\lambda}, \rho_{\lambda}\right)$ determines a projective structure $c_{\lambda}$ on $M$.

The following simple lemma is used to ensure convexity of the projective structures on Gromov-Thurston examples.

Let $H$ be a hyperplane in $\mathbb{R}^{n}$; let $H_{ \pm}$denote the closed half-spaces in $\mathbb{R}^{n}$ bounded by $H$. Suppose that $D \subset H_{-}$is a compact convex subset; let $F$ denote the intersection $H \cap D$. Pick a point $p \in H_{+}$. Let $\Sigma=\operatorname{Cone}_{p}(F)$ denote the (convex) cone with the vertex $p$ and the base $F$. 
Lemma 6.2 Suppose that for each $x \in D$ the segment $\overline{x p}$ crosses $H$ inside $F$. Then the union $D \cup \Sigma$ is convex.

Proof Under these assumptions, $D \cup \Sigma=\operatorname{Cone}(p, D)$. Now convexity follows from Lemma 2.1.

Suppose that $p_{i} \in H_{+}$is a sequence of points so that $D, H, p_{i}$ satisfy the conditions of Lemma 6.2. Assume that $\lim _{i} p_{i}=p \in \mathbb{R} P^{n} \backslash \mathbb{R}^{n}$. Let $\Sigma \subset \mathbb{R} P^{n}$ denote the limit of the cones Cone $p_{i}(F)$ with respect to the topology given by the Hausdorff distance between closed subsets of $\mathbb{R} P^{n}$. Let $E \subset H_{+}$be a compact convex subset contained in the cone $\Sigma$ so that

$$
D \cap E=D \cap H=E \cap H=F .
$$

Corollary 6.3 $D \cup E$ is convex.

Proof By taking the limit, Lemma 6.2 implies that for each pair of points $x_{1} \in \Sigma \backslash\{p\}$, $x_{2} \in D$, the intersection $\overline{x_{1} x_{2}} \cap H$ is contained in $F$. Since $E$ is a convex subset of $\Sigma$; it follows that $\overline{x_{1} x_{2}} \subset D \cup E$.

Let $P:=\operatorname{Span}(H)$.

Corollary 6.4 For each $t \in \mathbb{R}$, the union

$$
A_{P, p, t}(E) \cup D
$$

is convex.

Proof The set $E_{t}=A_{P, p, t}(E)$ is clearly convex. By the definition of $A_{P, p, t}$, the set $E_{t}$ is contained in the cone $\Sigma$ and $E_{t} \cap H=E_{t} \cap D=F$. Now the assertion follows from Corollary 6.3.

\section{Construction of convex projective structures on Gromov- Thurston manifolds}

Assume as before that the hyperbolic manifold $M$ satisfies Assumption 2 and let $M^{\prime}$ be the manifold constructed (using pieces of $M$ ) as in Section 3.

Theorem 7.1 For each natural number $m \geq 8$ which is divisible by 4 , the manifold $M^{\prime}$ admits a convex projective structure. 
Proof The proof breaks in two steps:

(1) We first bend the (hyperbolic) projective structure $c$ on the manifold with boundary $N^{\prime} \subset M^{\prime}$ (see Section 3) in order to obtain a new projective structure $c_{\lambda}$ which has flat boundary. We then construct a projective structure $a^{\prime}$ on $M^{\prime}$ by gluing two copies of $\left(N^{\prime}, c_{\lambda}\right)$ via an order 2 rotation. It is important to note here that in the following discussion we will regard $N^{\prime}$ as a submanifold of the original manifold $M$.

(2) We use the Theorem 4.3 to verify that $\left(M^{\prime}, a^{\prime}\right)$ is convex.

Step 1 Recall that our construction of the manifold $M^{\prime}$ starts with a choice of a "half" $N \subset M$ of the hyperbolic $n$-manifold $M$ and a fundamental domain $O \subset N$ Below we explain how to choose $O$. The boundary of $N$ is piecewise-geodesic, it is the union of two totally-geodesic submanifolds with boundary which we denote $L_{0}$ and $L_{5}$; the intersection $L_{0} \cap L_{5}=V$ is the common boundary of $L_{0}$ and $L_{5}$.

Since $m \geq 8$ is divisible by 4 , the dihedral group $D_{m}$ acting on $M$ contains the dihedral subgroup $D_{4}$. The group $D_{4}$ contains 4 reflections $\tau_{i}, i=1, \ldots, 4$, fixing the hypersurfaces

$$
\widehat{L}_{i} \subset M, i=1, \ldots, 4 .
$$

Whatever the choice of $N$ and $D_{4}$ is, we let

$$
L_{i}:=\widehat{L}_{i} \cap N, \quad i=1, \ldots, 4 \text {. }
$$

Given $N$, we choose the subgroup $D_{4} \subset D_{m}$ so that the angle between $L_{1}$ and $L_{0}$ is $\frac{\pi}{m}$ and then we take $O \subset N$ to be bounded by $L_{0}$ and $L_{1}$, see Figure 10 .

In order to define the manifold $N^{\prime}$ we delete the "piece" $O \subset N$ from $N$. Figure 11 depicts the resulting manifold $N^{\prime}$ (which we regard as a subset of $M$ ). The submanifolds with boundary $L_{2}, L_{3}, L_{4}$ separate $N^{\prime}$ into the submanifolds with corners $K_{1}, \ldots, K_{4}$, so that

$$
\partial K_{i}=L_{i} \cup L_{i+1}, i=1, \ldots, 4 .
$$

The angle between $L_{i}, L_{i+1}$ equals $\frac{\pi}{4}$, for $i=1, \ldots, 3$, and the angle between $L_{4}$ and $L_{5}$ is $\frac{\pi}{4}-\frac{\pi}{m}$.

The manifold $N^{\prime}$ is a submanifold with corners in the hyperbolic manifold $M$. We define a hyperbolic structure $h$ on $N^{\prime}$ as follows. Since $N^{\prime}$ is a convex subset of $M$, the universal cover $\tilde{N}^{\prime}$ of $N^{\prime}$ can be identified with a convex subset

$$
\tilde{N}^{\prime} \subset \tilde{M}=\dddot{H}^{n} .
$$




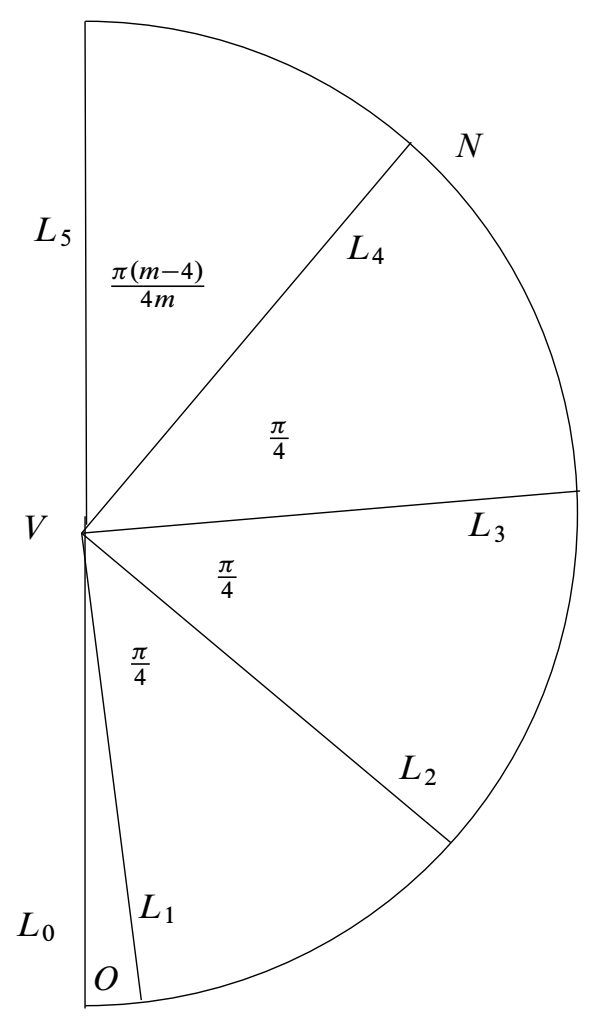

Figure 10: Submanifolds $L_{i} \subset N$.

We let dev: $\tilde{N}^{\prime} \rightarrow \mathbb{Q}^{n}$ denote the identity embedding. We have the corresponding embedding of the fundamental groups

$$
\iota: \pi_{1}\left(N^{\prime}\right) \rightarrow \pi_{1}(M) \subset \operatorname{Isom}\left(\mathbb{H}^{n}\right) .
$$

We regard dev and $\iota$ as the developing map and the holonomy representation respectively. They determine a hyperbolic structure $h$ on $N^{\prime}$. By using the projective model of $\mathbb{T}^{n}$, we regard $h$ as a projective structure $c$ on the manifold with boundary $N^{\prime}$. Note that the boundary of $N^{\prime}$ is not flat with respect to $c$, since it is bent along $V$. We will use a projective bending of $c$ in order to eliminate this singularity and obtain a new projective manifold $\left(N^{\prime}, c_{\lambda}\right)$ with flat boundary. In order to bend, we need a cooriented flat weighted hypersurface in $\left(N^{\prime}, c\right)$, which we define below.

Since each $L_{j}(j=1, \ldots, 4)$ is totally-geodesic (with respect to the hyperbolic structure $h)$, it is flat as a hypersurface in the projective manifold $\left(N^{\prime}, c\right)$. We define 


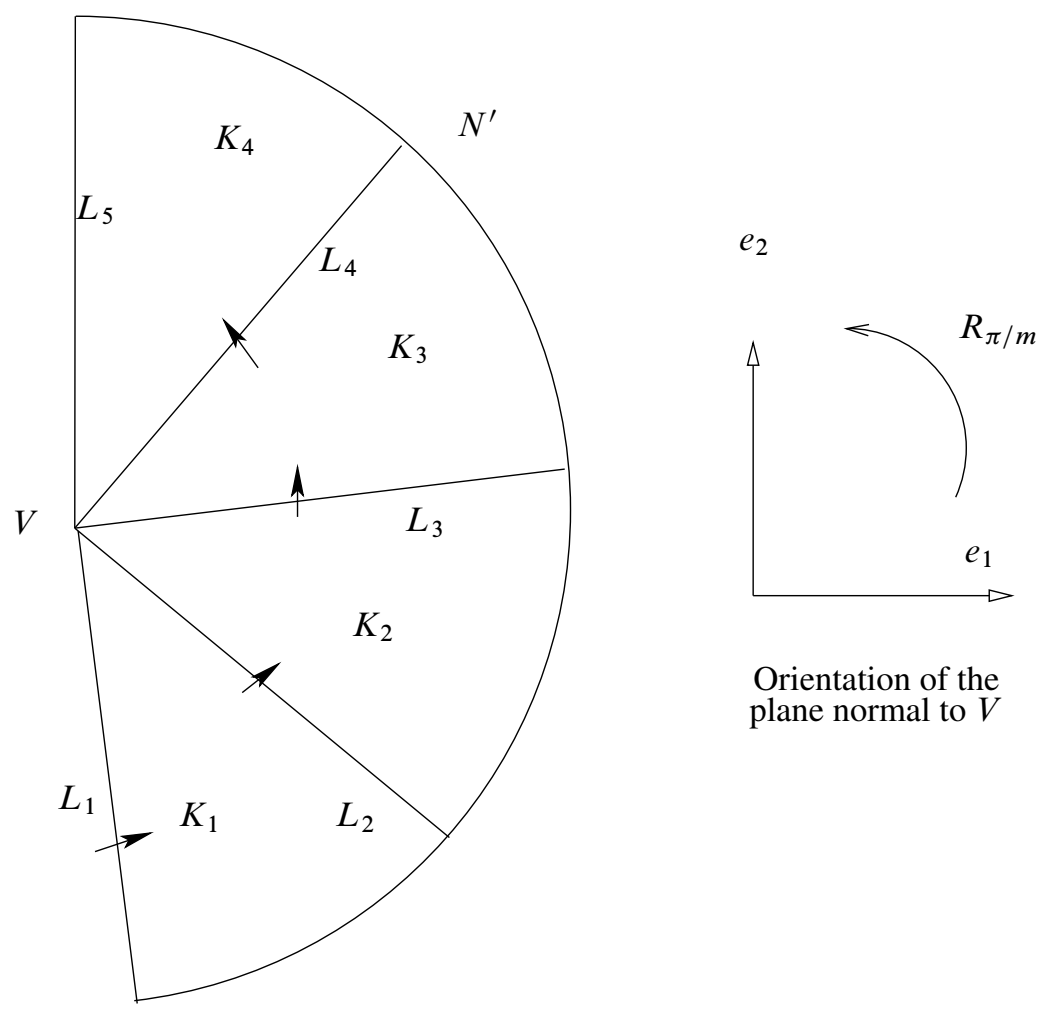

Figure 11: Coorientation on $L \subset N^{\prime}$.

coorientation for the hypersurface $L=L_{1} \cup \cdots \cup L_{4} \subset N^{\prime}$ by coorienting each $L_{j}$ 's as in Figure 11. (Each arrow indicates the direction from left to right.)

Our next goal is to define weights for the hypersurface $L$. We identify the hyperbolic space $\mathbb{T}^{n}$ with the unit ball $B$ in the Euclidean space $\mathbb{R}^{n} \subset \mathbb{R} P^{n}$, so that each flat hypersurface $L_{1}, \ldots, L_{4}, L_{5}$ corresponds (under the developing map) to a projective hyperplane $P_{1}, \ldots, P_{4}, P_{5} \subset \mathbb{R} P^{n}$ passing through the codimension 2 subspace $Q \subset$ $\mathbb{R} P^{n}$ containing the origin. The angles between $P_{i}, P_{i+1}$ are the same as the angles between $L_{i}, L_{i+1}, i=1, \ldots, 4$.

For each $i=1, \ldots, 4$ let $p_{i} \in \mathbb{R} P^{n} \backslash \mathbb{R}^{n}$ be the point dual to $P_{i}$ (see Section 5). We orient the 2-plane $Q^{\perp}$ normal to $Q$ in $\mathbb{R}^{n}$ as indicated in Figure 10. It remains to define the weight for $L$.

Since $m>4$, according to Section 5, there exist real numbers

$$
t_{1}, \quad t_{2}, \quad t_{3}=-t_{2}, \quad t_{4}=-t_{1}
$$


so that

$$
A_{P_{1}, p_{1}, t_{1}} \circ \cdots \circ A_{P_{4}, p_{4}, t_{4}}=R_{\frac{\pi}{m}},
$$

is the rotation around $Q$ by the angle $\frac{\pi}{m}$. Hence the rotation $R_{\frac{\pi}{m}}$ sends $P_{5}$ to $P_{1}$. (The order of the composition used here agrees with the orientation used for the 2-plane $Q^{\perp}$.)

Let $\mu$ denote the weight for $L$ defined by $L_{i} \mapsto e^{t_{i}}$. Let $\lambda:=(L, \mu)$ and $c_{\lambda}$ be the projective structure on $N^{\prime}$ obtained from $c$ by bending along $\lambda$.

Let $\tilde{L}_{i} \subset P_{i}, i=1, \ldots, 5$ be the images of the universal covers of the submanifolds $L_{i}$ under the (identity) developing map of $h$. Let $\widetilde{K}_{i} \subset \tilde{N}^{\prime}$, be the universal covers of the manifolds $K_{i}, i=1, \ldots, 4$, embedded in $\tilde{N}^{\prime}$ so that

$$
\tilde{K}_{i-1} \cap \tilde{K}_{i}=\tilde{L}_{i}, \quad i=2,3,4 .
$$

Then the developing map $\operatorname{dev}_{\lambda}$ of $c_{\lambda}$ satisfies:

$$
\begin{aligned}
& \operatorname{dev}_{\lambda} \mid \tilde{L}_{1}=\mathrm{Id}, \\
& \operatorname{dev}_{\lambda} \mid \tilde{K}_{1}=A_{P_{1}, p_{1}, t_{1}},
\end{aligned}
$$

and

$$
\operatorname{dev}_{\lambda} \mid \widetilde{K}_{4}=R_{\frac{\pi}{m}}
$$

see equation (8) in Section 6.

Set $c^{\prime}:=c_{\lambda}$. Since $R_{\frac{\pi}{m}}$ carries $P_{5}$ to $P_{1}$, the equation (10) implies that the projective manifold $\left(N^{\prime}, c^{\prime}\right)$ has flat boundary. Since the rotation $R_{\frac{\pi}{m}}$ belongs to the isometry group of $\mathbb{Q}^{n}$, the restriction of the projective structure $c^{\prime}$ to $\partial N^{\prime}$ is a hyperbolic structure, necessarily isometric to the hyperbolic metric on the totally-geodesic boundary of the hyperbolic manifold $(N, h)$. The image of the developing map of $c^{\prime} \mid \partial N^{\prime}$ is the hyperbolic hyperplane $H_{1}:=P_{1} \cap \mathbb{\boxplus}^{n}$.

Let

$$
\theta^{\prime}:\left(\partial N^{\prime}, c^{\prime}\right) \rightarrow\left(\partial N^{\prime}, c^{\prime}\right)
$$

denote the isometric involution which interchanges $L_{1}$ and $L_{5}$ (see Section 3). This involution corresponds to the order 2 rotation around $Q$ in $\mathbb{R} P^{n}$. Thus we obtain a projective manifold with flat boundary $\left(N^{\prime}, c^{\prime}\right)$ and the order 2 automorphism $\theta^{\prime}$ of its boundary.

We next glue two copies of $\left(N^{\prime}, c^{\prime}\right)$ via $\theta^{\prime}$. It is done as follows. Recall that the developing map of $c^{\prime} \mid \partial N^{\prime}$ is a diffeomorphism to the hyperbolic hyperplane $H_{1} \subset \mathbb{M ^ { n }}$. 
Since $\partial N^{\prime}$ is compact, there exists a regular neighborhood $T$ of $\partial N^{\prime}$ in $N^{\prime}$, so that the developing map of $c^{\prime} \mid T$ is a diffeomorphism

$$
\operatorname{dev}_{\lambda}: \widetilde{T} \rightarrow \operatorname{dev}_{\lambda}(\widetilde{T}) \subset \mathbb{M}^{n},
$$

to a neighborhood of $H_{1}$ in one of the half-spaces in $\mathbb{\boxplus}^{n}$ determined by $H_{1}$. Hence the restriction of $c^{\prime}$ to $T$ is a hyperbolic structure.

Let $\left(N_{ \pm}^{\prime}, c_{ \pm}^{\prime}\right)$ denote the two copies of $\left(N^{\prime}, c^{\prime}\right)$ :

$$
N_{ \pm}^{\prime}=N^{\prime} \times\{ \pm 1\}
$$

The disjoint union

$$
\left(\widehat{N}, \widehat{c}^{\prime}\right)=\left(N_{+}^{\prime}, c_{+}^{\prime}\right) \sqcup\left(N_{-}^{\prime}, c_{-}^{\prime}\right)
$$

is also a projective manifold with flat boundary; the automorphism $\theta^{\prime}$ determines an automorphism

$$
\hat{\theta}^{\prime}:\left(\widehat{N}, \widehat{c}^{\prime}\right) \rightarrow\left(\widehat{N}, \widehat{c}^{\prime}\right)
$$

which interchanges $\partial N_{-}^{\prime}$ and $\partial N_{+}^{\prime}$. Set

$$
M^{\prime}=\widehat{N} / \widehat{\theta}=N_{+}^{\prime} \cup_{\theta^{\prime}} N_{-}^{\prime}
$$

Let $D \subset M^{\prime}$ be the hypersurface which is the projection of $\partial \hat{N}$.

The projective structure on $M^{\prime} \backslash D$ is the restriction of the projective structures $c_{ \pm}^{\prime}$. It remains to define the projective structure on $M^{\prime}$ near $D$. Observe that

$$
T^{\prime}:=T_{+} \cup_{\theta^{\prime}} T_{-}=T \times\{+1\} \cup_{\theta^{\prime}} T \times\{-1\} \subset M^{\prime}
$$

is a neighborhood of $D$. The projective structures $c_{ \pm}^{\prime} \mid T_{ \pm}$are hyperbolic with geodesic boundary and $\theta^{\prime}: \partial T \rightarrow \partial T$ is a hyperbolic isometry. Therefore we get the induced hyperbolic structure on $T^{\prime}$. This hyperbolic structure determines a projective structure which agrees with

$$
c_{ \pm}^{\prime} \mid \operatorname{int}\left(N_{ \pm}^{\prime}\right)
$$

As the result we obtain the projective structure $a^{\prime}$ on the manifold $M^{\prime}$. By construction, the manifold $\left(M^{\prime}, a^{\prime}\right)$ admits an order 2 automorphism $\theta$ which fixes the codimension 2 submanifold $V^{\prime}$ pointwise and corresponds (under the developing map) to the order 2 rotation in $\mathbb{R} P^{n}$. Here $V^{\prime} \subset M^{\prime}$ is the submanifold corresponding to $V \subset N \subset M$.

This concludes Step 1.

Step 2 Let $N_{ \pm}^{\prime}$ denote the two copies of $N^{\prime}$ used to construct $M^{\prime}$. According to our discussion in Section 3, the hyperbolic manifold $\left(N^{\prime}, c\right)$ is tiled by $m-1$ isometric copies $O_{j}$ of the fundamental domain $O$ of the group $D_{m} \subset \operatorname{Aut}(M)$ (see Section 3). The intersection $\cap_{j} O_{j}$ is the codimension 2 totally-geodesic submanifold $V \subset N^{\prime}$. 
Let $W_{1}^{+}=L_{1}, W_{1}^{+}, \ldots, W_{m}^{+} \subset N_{+}^{\prime}$ denote the flat hypersurfaces which appear as the boundary components of the domains $O_{j} \backslash V$. For each $j$ let $W_{j}^{-}:=\theta\left(W_{j}^{+}\right) \subset M^{\prime}$. Then for each $j$, the flat hypersurfaces with boundary $W_{j}^{-}, W_{j}^{+}$match in $M^{\prime}$ to form a flat hypersurface (without boundary) $S_{j} \subset\left(M^{\prime}, a^{\prime}\right)$ which is invariant under the automorphism $\theta:\left(M^{\prime}, a^{\prime}\right) \rightarrow\left(M^{\prime}, a^{\prime}\right)$.

The flat hypersurfaces $S_{j}$ cut the projective manifold $\left(M^{\prime}, a^{\prime}\right)$ into components, each of which is (projectively) isomorphic to the convex hyperbolic manifold with corners $O$.

We now pass to the universal cover $X=\left(\tilde{M}^{\prime}, \tilde{a}^{\prime}\right)$ of $\left(M^{\prime}, a^{\prime}\right)$. The codimension 2 submanifold $V^{\prime}$ lifts to $X$ to a disjoint union of codimension two submanifolds, each of which is isomorphic to the open $(n-2)$-disk.

The inverse image $\widetilde{S} \subset X$ of $S:=\cup_{j} S_{j}$ is a union of flat hypersurfaces in $X$ (walls) which intersect along the codimension 2 submanifolds above. The closure $C_{j}^{\prime}$ of each component of $X \backslash \widetilde{S}$ is a convex subset, which is projectively isomorphic to the universal cover of the hyperbolic manifold with corners $O$. Thus we obtain a stratification of $X$ by closed subsets which are:

(1) Codimension 0 strata (cells): Convex sets $C_{j}^{\prime}$.

(2) Codimension 1 strata: Facets $F_{i}^{\prime} \subset C_{j}^{\prime}$, which are $(n-1)$-dimensional intersections of $C_{j}^{\prime}$ with walls.

(3) Codimension 2 strata: Components of the preimage of $V \subset M^{\prime}$.

The dual complex for this stratification of $X$ is a regular 2-dimensional cell complex $Z$, where every 2 -cell has $2(m-1) \geq 14$ edges. By convexity, the links of vertices of $Z$ do not contain any bigons. Thus $Z$ satisfies the $C^{\prime}(1 / 14)$ (and, hence, $\left.C^{\prime}(1 / 7)\right)$ small cancellation condition.

We now analyze the unions of the adjacent cells. Suppose that $C_{1}^{\prime}, C_{2}^{\prime}$ share a facet $F^{\prime}$.

Lemma 7.2 $C_{1}^{\prime} \cup C_{2}^{\prime}$ is isomorphic to a proper convex subset of $\mathbb{R} P^{n}$.

Proof Let dev: $X \rightarrow \mathbb{R} P^{n}$ denote the developing map of $\left(M^{\prime}, a^{\prime}\right)$ and set $B_{i}^{\prime}:=$ $\operatorname{dev}\left(C_{i}^{\prime}\right), i=1,2$.

Recall that the projective structure $a^{\prime}$ on $M^{\prime}$ is obtained by bending of the hyperbolic structure on $M$. Therefore $C_{1}^{\prime} \cup C_{2}^{\prime}$ is isomorphic to the projective manifold that can be described as follows. Consider the compact manifolds with corners $O_{1}, O_{2} \subset M$; they intersect along a hypersurface $W:=W_{1}^{+}$. Lift these manifolds to simply-connected regions $\widetilde{O}_{1}, \widetilde{O}_{2} \subset \widetilde{M}$, which share a hypersurface $\tilde{W}$, which passes through the origin. 
Let $P:=\operatorname{Span}(\tilde{W})$, and $p \in \mathbb{R} P^{n} \backslash \mathbb{R}^{n}$ be the point dual to $P$. Clearly, $O_{1} \cup O_{2}$ is convex; therefore $U:=\widetilde{O}_{1} \cup \widetilde{O}_{2}$ is convex as well. Note that there exists a Euclidean reflection $\tau$ in the hyperplane $H$ preserves the union $U$ and interchanges $\widetilde{O}$ and $\widetilde{O}_{2}$. Then, by appropriately normalizing the developing map dev of $\left(M^{\prime}, a^{\prime}\right)$, we conclude that

$$
\begin{aligned}
& \operatorname{dev}=\mathrm{Id}: \widetilde{O}_{1} \rightarrow B_{1}^{\prime}, \\
& \operatorname{dev}=A_{P, p, t}: \widetilde{O}_{2} \rightarrow B_{2}^{\prime},
\end{aligned}
$$

and

$$
\operatorname{dev}\left(F^{\prime}\right)=\tilde{W},
$$

where $t$ depends on the pair $\left(C_{1}^{\prime}, C_{2}^{\prime}\right)$.

We are now ready to prove convexity of $B_{1}^{\prime} \cup B_{2}^{\prime}$. Suppose that there exists a point $x_{1} \in B_{1}^{\prime}$ such that the projective line $l$ through $x_{1}, p$ crosses $P$ at a point $y$ which is not in $\widetilde{W}$. Then, by symmetry, $l \cap \widetilde{O}_{2}$ contains a point $x_{2}=\tau\left(x_{1}\right)$ and the segment $\overline{x_{1} x_{2}}$ contains a point $y \notin U$. This contradicts convexity of $U$.

Therefore we can apply Corollary 6.3 to $B_{1}^{\prime} \cup B_{2}^{\prime}$ (with $D:=\widetilde{O}_{1}, E:=\widetilde{O}_{2}$ ) and conclude that $B_{1}^{\prime} \cup B_{2}^{\prime}$ is convex.

We now de-projectivize the projective manifold $X$. We replace each cell $C_{j}^{\prime}$ with a convex cone $\hat{C}_{j}^{\prime}$, etc. The result is an affine $(n+1)$-manifold $\hat{X}$ which is obtained by gluing the convex cones $\widehat{C}_{j}^{\prime}$. We next observe that the conditions of Theorem 4.3 are satisfied by $\hat{X}$. For instance, Parts 1 and 2 of Assumption 3, follow from the fact that the cells $C_{j}^{\prime}$ are isomorphic to the universal cover $\widetilde{O}_{1}$ with the codimension 1 and 2 faces of $C_{j}$ covering strata of $\partial O_{1}$. Parts 3,4 , and 5 follow from the fact that the limit sets of the faces correspond to the limit sets of their stabilizers in Isom $\left(\mathbb{M}^{n}\right)$. Part 1 of Assumption 4, comes from the fact that the manifold $\left(M^{\prime}, a^{\prime}\right)$ is obtained by gluing the boundaries of two copies of $\left(N^{\prime}, c_{\lambda}\right)$ via an order 2 rotation. Part 2 of Assumption 4 follows from Lemma 7.2.

Therefore Theorem 4.3 implies that $\tilde{X}$ is isomorphic to a proper homogeneous open convex cone in $\mathbb{R}^{n+1}$. Thus $X$ is isomorphic to a proper open convex subset of $\mathbb{R} P^{n}$ and therefore the manifold $M^{\prime}$ is a convex projective manifold. This concludes the proof of Theorem 7.1.

Corollary 7.3 The projective manifold $M^{\prime}$ is strictly convex.

Proof Since $M^{\prime}$ admits a metric of negative curvature, its fundamental groups are Gromov-hyperbolic. Since $M^{\prime}$ is convex, it is strictly convex by Theorem 1.2. 


\section{Appendix A Dual complex to the conical stratification of an affine manifold}

The material of this section is standard, we present it for the sake of completeness. Let $X$ be an affine manifold which is the union of cones $C_{j}^{\prime}, j \in J$, and let $Z$ be the cell complex dual to the stratification of $X$, see Section 4. We give $Z$ the weak topology. The main goal of this appendix is to prove the following proposition.

Proposition A.1 $Z$ is homotopy-equivalent to $X$.

Proof We define the barycentric subdivision $\beta(Z)$ of $Z$ as follows. First, take the barycentric subdivision $\beta(T)$ of the 1-skeleton $T$ of $Z$. Next, for each 2-cell $c$ in $Z$, pick an interior point $o_{c} \in c$. Now cone off the boundary circle $\partial c$ (regarded as a subcomplex of $\beta(Z))$ from the point $o_{c}$. The result is the barycentric subdivision of $c$. Repeat this procedure for each 2-cell $c$. The resulting complex $\beta(Z)$ is the simplicial complex associated with the poset whose elements are the cells of $Z$. The geometric realization of $\beta(Z)$ is, of course, the same as the geometric realization of $Z$.

Our goal is to promote the stratification of $X$ by the convex cones of codimension 0,1 and 2 into an open covering by contractible sets with contractible intersections, so that the nerve of the resulting covering is $\beta(Z)$.

We begin by triangulating the space $X$ so that each simplex in the triangulation is linear with respect to the affine structure on $X$ and so that each stratum in $X$ is a union of simplices in the resulting simplicial complex $\delta(X)$.

A priori, stars of the disjoint strata in $X$ (with respect to the above triangulation) are not disjoint. To correct this, take the second barycentric subdivision $Y$ of $\delta(X)$. Then disjoint strata in $X$ have disjoint stars with respect to $Y$. Given a stratum $S$ in $X$ let $\operatorname{st}(S)$ denote the star of $S$ in $Y$. Let $Y_{S}$ denote the open star of $S$ in $Y$, ie

$$
Y_{S}:=\operatorname{st}(S) \backslash \bigcup_{\sigma \in \Sigma} \sigma
$$

where $\Sigma$ consists of all simplices in $Y$ which are disjoint from $S$.

We obtain an open covering $\mathcal{U}$ of $X$ by the open stars $Y_{S}$ of the strata of $X$. Since disjoint strata have disjoint stars, whenever

$$
Y_{S_{1}} \cap Y_{S_{2}} \cap Y_{S_{3}} \neq \varnothing
$$

after renumbering the strata $S_{i}$, we obtain

$$
S_{1} \subset S_{2} \subset S_{3} .
$$


Therefore the nerve of the covering $\mathcal{U}$ is isomorphic to the poset whose elements are the closed strata $S \subset X$, ie to the simplicial complex $\beta(Z)$. Moreover, every nonempty intersection

$$
Y_{S_{1}} \cap Y_{S_{2}} \cap Y_{S_{3}}
$$

has to be equal to $Y_{S_{1}}$ since

$$
Y_{S_{1}} \subset Y_{S_{2}} \subset Y_{S_{3}} .
$$

Since $S_{1}$ is contractible, it follows that the open star $Y_{S_{1}}$ is contractible as well. Then the nerve $\beta(Z)$ of the covering $\mathcal{U}$ is is homotopy-equivalent to $X$. Therefore $Z$ is also homotopy-equivalent to $X$.

Corollary A.2 $Z$ is simply-connected.

Proof Since $X$ is simply-connected, it follows that $Z$ is simply-connected as well.

\section{References}

[1] A F Beardon, The geometry of discrete groups, Graduate Texts in Mathematics 91, Springer, New York (1983) MR698777

[2] Y Benoist, Convexes divisibles I, from: "Algebraic groups and arithmetic", Tata Inst. Fund. Res., Mumbai (2004) 339-374 MR2094116

[3] Y Benoist, Convexes hyperboliques et quasiisométries, Geom. Dedicata 122 (2006) 109-134 MR2295544

[4] M R Bridson, A Haefliger, Metric spaces of non-positive curvature, Grundlehren der Mathematischen Wissenschaften 319, Springer, Berlin (1999) MR1744486

[5] S Choi, W M Goldman, The deformation spaces of convex $\mathbb{R} \mathbb{P}^{2}$-structures on 2orbifolds, Amer. J. Math. 127 (2005) 1019-1102 MR2170138

[6] CH Dowker, Topology of metric complexes, Amer. J. Math. 74 (1952) 555-577 MR0048020

[7] E Ghys, P de la Harpe, Sur les groupes hyperboliques d'après Mikhael Gromov, Progress in Mathematics 83, Birkhäuser, Boston (1990) MR1086648

[8] W Goldman, Lecture notes of a course on affine and projective structures on manifolds (1988) University of Maryland

[9] W M Goldman, Convex real projective structures on compact surfaces, J. Differential Geom. 31 (1990) 791-845 MR1053346

[10] M Gromov, W Thurston, Pinching constants for hyperbolic manifolds, Invent. Math. 89 (1987) 1-12 MR892185 
[11] M Kapovich, Kleinian groups in higher dimensions (2006) To appear in Proceedings of A Reznikov Memorial Conference "Geometry and Dynamics of Groups and Spaces", Bonn

[12] M Kapovich, J J Millson, Bending deformations of representations of fundamental groups of complexes of groups (1996) preprint

[13] M Kapovich, J J Millson, Hodge theory and the art of paper folding, Publ. Res. Inst. Math. Sci. 33 (1997) 1-31 MR1442490

[14] J Lee, A convexity theorem for real projective structures arXiv:0705.3920

[15] J Maubon, Variations d'entropies et déformations de structures conformes plates sur les variétés hyperboliques compactes, Ergodic Theory Dynam. Systems 20 (2000) 1735-1748 MR1804955

[16] P Susskind, G A Swarup, Limit sets of geometrically finite hyperbolic groups, Amer. J. Math. 114 (1992) 233-250 MR1156565

[17] È B Vinberg, Discrete linear groups that are generated by reflections, Izv. Akad. Nauk SSSR Ser. Mat. 35 (1971) 1072-1112 MR0302779

Department of Mathematics, University of California,

Davis, CA 95616, USA

kapovich@math.ucdavis.edu

http://www . math.ucdavis.edu/ kapovich/

Proposed: Walter Neumann

Received: 8th June 2006

Seconded: Martin Bridson and Benson Farb

Revised: 17 August 2007 